INDEPENDENT JOURNAL OF MANAGEMENT \& PRODUCTION (IJM\&P)

\title{
MULTICRITERIA HYBRID FLOW SHOP SCHEDULING PROBLEM: LITERATURE REVIEW, ANALYSIS, AND FUTURE RESEARCH
}

\author{
Márcia de Fátima Morais \\ State University of Paraná (UNESPAR), Brazil \\ E-mail:marciafmorais@yahoo.com.br \\ Thays Josyane Perassoli Boiko \\ State University of Paraná (UNESPAR), Brazil \\ E-mail: thaysaperssoli@bol.com.br \\ Leandro dos Santos Coelho \\ Federal University of Parana (UFPR), Brazil \\ E-mail: Iscoelho2009@gmail.com \\ Rony Peterson da Rocha \\ State University of Paraná (UNESPAR), Brazil \\ E-mail: ronypeterson_eng@hotmail.com \\ Paulo Roberto Paraíso \\ State University of Maringá (UEM), Brazil \\ E-mail:paulo@deq.uem.br \\ Submission: 11/06/2014 \\ Accept: 25/06/2014
}

\section{ABSTRACT}

This research focuses on the Hybrid Flow Shop production scheduling problem, which is one of the most difficult problems to solve. The literature points to several studies that focus the Hybrid Flow Shop scheduling problem with monocriteria functions. Despite of the fact that, many real world problems involve several objective functions, they can often compete and conflict, leading researchers to concentrate their efforts on the development of methods that take this variant into consideration. The goal of the study is to review and analyze the methods in order to solve the Hybrid Flow Shop production scheduling problem with multicriteria functions in the literature. The analyses were performed using several papers that have been published over the years, also the parallel machines types, the approach used to develop solution methods, the type of method develop, the objective function, 
the performance criterion adopted, and the additional constraints considered. The results of the reviewing and analysis of 46 papers showed opportunities for future research on this topic, including the following: (i) use uniform and dedicated parallel machines, (ii) use exact and metaheuristics approaches, (iv) develop lower and uppers bounds, relations of dominance and different search strategies to improve the computational time of the exact methods, ( $v$ ) develop other types of metaheuristic, (vi) work with anticipatory setups, and (vii) add constraints faced by the production systems itself.

Keywords: Production Scheduling; Multicriteria Functions; Hybrid Flow Shop; Literature.

\section{INTRODUCTION}

Production scheduling is one of the most complex activities in the management of production systems. It is closely connected with the firm's performance in terms of speed, reliability, flexibility, quality, and cost.

The theory of production scheduling, that aims to provide guidelines and methods, for efficient use of resources, has been the subject of countless papers, over the past five decades (MORAIS; MOCCELLIN, 2010). Although several features of scheduling problems are still underexplored due to the variety of production environments, the available resources, restrictions may be imposed and there are multiple objectives to be achieved.

Therefore, this research aims to identify and quantify the published papers that present solution methods for scheduling problems in Hybrid Flow Shop with multicriteria. The results, of this research, may be useful for future research, towards the development of new solution methods, and/or for the application of methods investigated in the context of real companies, with this kind of scheduling problem.

In this article, the term multicriteria is used generically to mean two or more criteria (bicriteria, tricriteria, and multicriteria), which are processed simultaneously in the same objective function.

It is noted that this research is dedicated solely to the production scheduling of jobs, not dealing with the production scheduling of batches of jobs. 
INDEPENDENT JOURNAL OF MANAGEMENT \& PRODUCTION (IJM\&P)

This paper is structured in six sections. After the presentation of the context and research objectives, the theoretical framework is explained in Section 2. In Section 3, the research methodology is presented. Then, the papers that present solution methods for the multicriteria Hybrid Flow Shop scheduling problem are cited. An analysis of papers is presented in Section 5; followed by conclusions, and final considerations, in the sixth section.

\section{THEORETICAL FRAMEWORK}

\subsection{Production Scheduling Problem}

Production scheduling is one of the activities of the Planning, Programming and Production Control. This is responsible for deciding the allocation of resources (called machines) over time to perform individual items (jobs and/or batch of jobs, called jobs), in order to better meet a predefined set of criteria (BAKER, 1974, MACCARTHY; LIU, 1993, YANG; LIAO, 1999, and PINEDO, 2008).

One can understand the production scheduling as a set of functions of decision-making, involving: i) allocation decisions machines to process jobs over time (Baker, 1974), called schedule (PINEDO, 1995); ii) decisions sequencing jobs (Baker, 1974), called sequence, which correspond to the order in which jobs are processed on a given machine (PINEDO, 1995).

Therefore, a scheduling problem is "a problem of $n$ jobs $\left\{\mathrm{J}_{1}, \mathrm{~J}_{2}, \ldots, \mathrm{J}_{\mathrm{j}}, \ldots, \mathrm{J}_{n}\right\}$ that must be processed on $m$ machines available $\left\{M_{1}, M_{2}, \ldots, M_{k}, \ldots, M_{m}\right\}$ " (FRENCH, 1982, p. 5). I.e., a scheduling problem "...consists of determining the order or sequence in which the machines will process the jobs so as to optimize some measure of performance" (JOHNSON; MONTGOMERY, 1974, p.322).

Due to the complexity related to obtaining and maintaining production schedules in firms, this activity is a major obstacle in the search for a good performance of production processes. In fact, the scheduling problem is among the most difficult problems of resolution (MORAIS; MOCCELLIN, 2010).

Based on MacCarthy; Liu (1993), Allahverdi; Cheng; Kovalyov (2008), and Pinedo (2008), lists the following types of scheduling environments: single machine; parallel machine; flow shop; permutational flow shop; job shop; hybrid flow shop or 
INDEPENDENT JOURNAL OF MANAGEMENT \& PRODUCTION (IJM\&P)

http://www.ijmp.jor.br

v. 5, n. 4, October - December 2014

ISSN: 2236-269X

DOI: 10.14807/ijmp.v5i4.242

flow shop with multiple machines; hybrid job shop or job shop with multiple machines, and; open shop. Figure 1 presents the relationship between these environments.

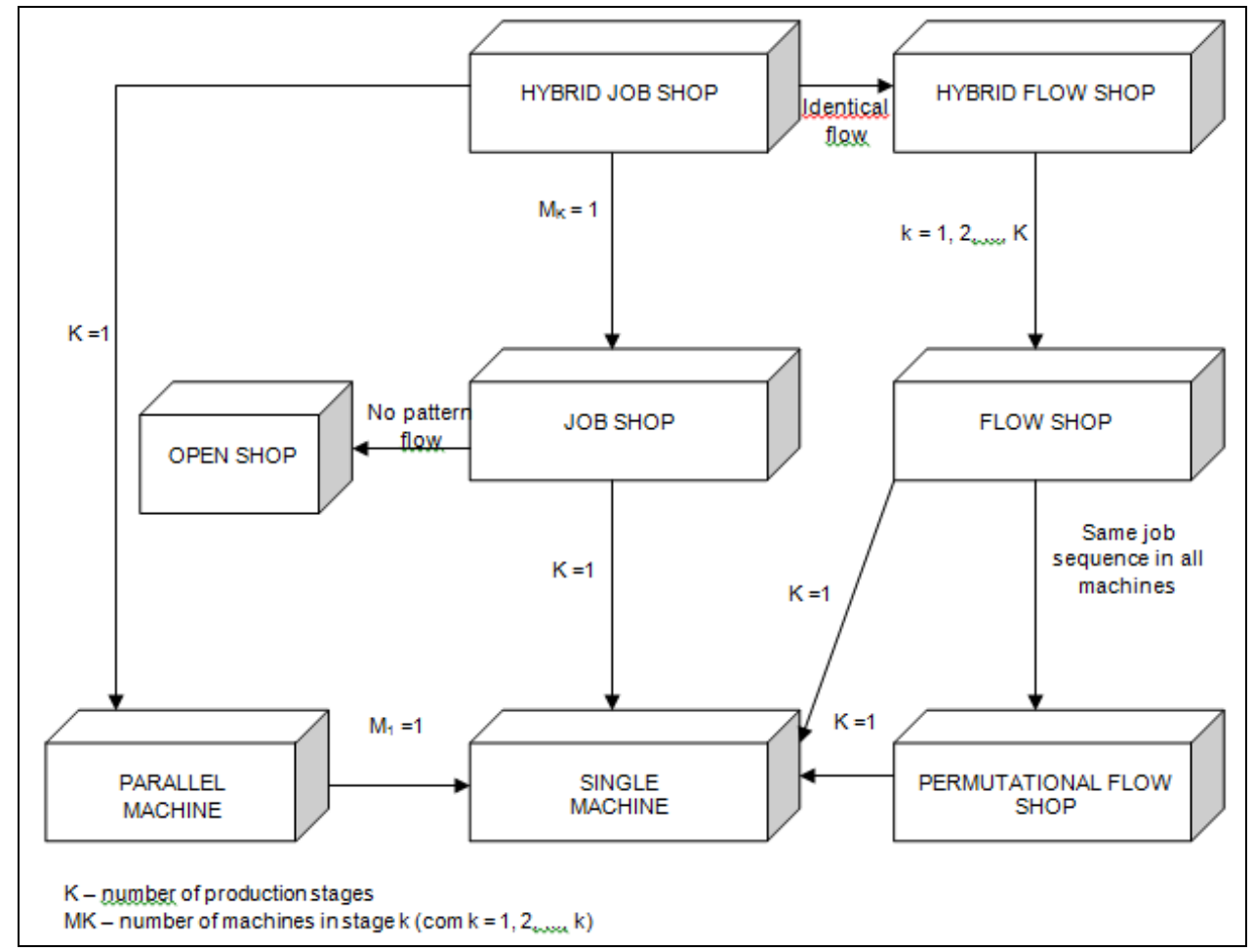

Figure 1: Environments of scheduling and their relationships

Source: MacCarthy; Liu (1993)

This research is dedicated to the Hybrid Flow Shop.

\subsection{Hybrid Flow Shop}

It is difficult to find a general definition for Hybrid Flow Shop; however, for the purposes of this research, presented by Sethanan (2001) the following is appropriated.

The Hybrid Flow Shop is a type of flow shop in which, at least one of the $k$ stages of production, the number of machines is greater than $1(\mathrm{k}<\mathrm{m})$. In the stages that the number of machines is greater than 1 , there are $k$ machines or processors in parallel, and each jobs is processed on only one machine stage (SETHANAN, 2001). Figure 2 illustrates the Hybrid Flow Shop scheduling environment.

According to Burtseva; Yaurima; Parra (2010), the possible machine set environments in stage $i$ of a Hybrid Flow Shop are the following: 
- Identical (ID) machines in parallel: the $m_{i}$ machines in the set have the same speed; therefore, job $j$ may be processed on any of $m_{i}$ machines, since the job processing time is the same for all machines;

- Uniformed (UN) machines in parallel: the $m_{i}$ machines in the set have different speeds; a job $j$ may be processed on any machine of a set; however, its processing time is proportional to the machine speed;

- Unrelated (UR) machines in parallel: the $m_{i}$ machines in the set have different speeds; a job $j$ may be processed on any machine of a set; however, its processing time, reveals not to be proportional to the speed of the machine;

- Dedicated (DED) machines in parallel: the $m_{i}$ machines in the set are dedicated to perform specific jobs, performing specific jobs.

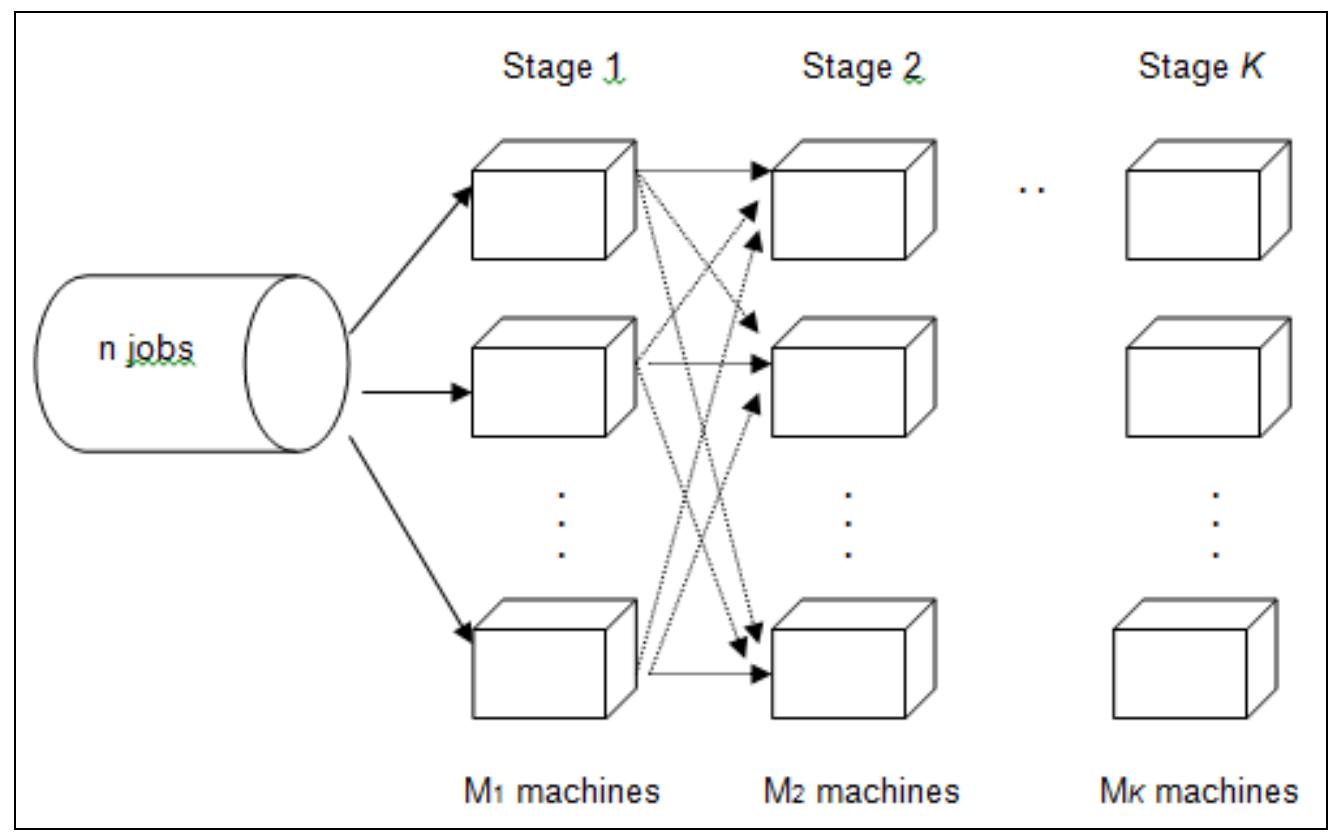

Figure 2: Hybrid Flow Shop scheduling environment Source: Morais (2008)

\subsection{Performance Criteria in Scheduling Problems}

The production scheduling is always carried out in order to reach a criterion or set a performance criteria that characterize the nature of the scheduling problem (BOIKO; MORAIS, 2009). Based on French (1982), Bedworth; Bailey (1987), MacCarthy; Liu (1993), Morton; Pentico (1993), and Pinedo (2008), the Table 1 presents the performance criteria, adopted in scheduling problems.

These different performance criteria, according to Baker (1974), relate to three types of decision-making: 
INDEPENDENT JOURNAL OF MANAGEMENT \& PRODUCTION (IJM\&P)

http://www.ijmp.jor.br

v. 5 , n. 4 , October - December 2014

ISSN: 2236-269X

DOI: 10.14807/ijmp.v5i4.242

i) Efficient use of resources;

ii) Rapid response to demand, and;

iii) Adaptation to prescribed deadlines of a job that, if reached, cancels the processing that has already been accomplished.

Table 1: Performance Criteria adopted in scheduling problems

\begin{tabular}{|c|c|}
\hline Notation & Description \\
\hline$C_{j}$ & Completion Time of Job \\
\hline $\mathrm{C}_{\max }$ & Makespan \\
\hline$\sum C_{i} / n$ & Mean Completion Time \\
\hline$\sum C_{j}$ & Completion Time \\
\hline$\sum w_{j} C_{j}$ & Weighted Completion Time \\
\hline$E_{j}$ & Earliness of JobTotal \\
\hline$\sum E_{j}$ & Total Earliness \\
\hline$E_{\max }$ & Earliness Maximum \\
\hline$\sum E j / n$ & Mean Earliness \\
\hline$\sum w_{j} E_{j}$ & Weighted Total Earliness \\
\hline$F_{j}$ & Flow Time of Job \\
\hline$\sum F_{j} / n$ & Mean Flow Time \\
\hline$\sum w_{j} F_{j}$ & Weighted Total Flow Time \\
\hline$\sum \mathrm{Lj}$ & Total Lateness \\
\hline $\mathrm{L}_{\mathrm{j}}$ & Lateness of Job \\
\hline $\mathrm{L}_{\max }$ & Lateness Maximum \\
\hline$\sum L_{j} / n$ & Mean Lateness \\
\hline$\sum w_{j} L_{j}$ & Weighted Total Lateness \\
\hline$T_{j}$ & Tardiness of Job \\
\hline$\sum T_{j}$ & Total Tardiness \\
\hline $\mathrm{T}_{\max }$ & Tardiness Maximum \\
\hline$\sum T_{j} / n$ & Mean Tardiness \\
\hline$\sum w_{j} T_{j}$ & Weighted Total Tardiness \\
\hline$\sum \mathrm{U}_{\mathrm{j}}$ & Number of Late Jobs \\
\hline$\sum U_{j} / n$ & Mean Number of Late Jobs \\
\hline$W_{j}$ & Time to Wait \\
\hline$\Sigma W_{j}$ & Total Time to Wait \\
\hline $\mathrm{W}_{\max }$ & Wait Time Maximum \\
\hline$\sum W_{j} / n$ & Mean Time to Wait \\
\hline$\sum w_{j} w_{j}$ & Weighted Total Time to Wait \\
\hline
\end{tabular}


INDEPENDENT JOURNAL OF MANAGEMENT \& PRODUCTION (IJM\&P)

Regarding the optimization criteria Lateness and Tardiness, Pinedo (2008) explains the delay of job $j$ (Lateness $-L_{j}$ ) the difference between the completion time of job and its due date, is defined as $L_{j}=C_{j}-d_{j}$, while the delay of job $j$ (Tardiness $\mathrm{T}_{\mathrm{j}}$ ) corresponds to the delay in completing the job in relation to its due date. According to Pinedo (2008) Tardines of job is defined as $T_{j}=\max \left(C_{j}-d_{j}, 0\right)=\max \left(L_{j}\right.$, $0)$. The difference between Tardiness and Lateness lays on the fact that Tardiness is never negative.

In addition to performance criteria, mentioned above, according to Godinho Filho et al. (2013) other criterias have been reported in the literature: Due Date Cost $\left(\Sigma c_{j} d_{j}\right)$; Bottleneck Utilization Rate (BTK); Capacity Utilization Rate (CPT); Inventory Costs (IC); Number of Families (NF); Overtime (OT); Size Buffer (SB); Time Blocking of the Machines (MBT); Total Cost of Opportunity (TCO); Total Cost of Setup (TSC); Total Cost Utility (TCU); Total Idle Time (MIT); Total Setup Time (TST ou $\Sigma \mathrm{Sj}$ ); Transportation Costs (TC); Work In Process (WIP).

\subsection{Constraints in Scheduling Problems}

Regarding to the assumptions of scheduling problems, these can be divided into hypotheses about jobs and/or job groups, about machinery and policy operations (GUPTA; STAFFORD, JR., 2006). These assumptions determine the constraints of a specific scheduling problem, in order to make the problem as similar as possible to a real situation.

Based on Allahverdi et al. (1999), Allahverdi et al (2008), and Pinedo (2008), Table 2 presents the constraints that may be incorporated in scheduling problems; as well as the notation adopted in this article to describe these constraints.

In addition to these constraints, single machine is a special case in which many stages (not all) in a Hybrid Flow Shop can have only one machine (BURTSEVA; YAURIMA; PARRA, 2010); this environment is termed as Hybrid Flow Shop with a dominant stage (MORAIS; GODINHO FILHO; BOIKO, 2013). 
INDEPENDENT JOURNAL OF MANAGEMENT \& PRODUCTION (IJM\&P)

http://www.ijmp.jor.br

v. 5 , n. 4, October - December 2014

ISSN: 2236-269X

DOI: 10.14807/ijmp.v5i4.242

Table 2: Constraints incorporated in scheduling problems.

\begin{tabular}{|c|c|}
\hline Notation & Description \\
\hline Batch & Batch jobs \\
\hline Bfr & No intermediate buffers \\
\hline Bkdwn & Breakdown \\
\hline Block & Blocking \\
\hline Btlck & Bottleneck resource \\
\hline Com_d & Common due dates \\
\hline Diff_d & Different due dates \\
\hline Diff_r $r_{j}$ & Different release dates \\
\hline Dyn_arr & Dynamics arrivals of jobs \\
\hline H_Fin & Finite planning horizon \\
\hline L_WT & Limited waiting time \\
\hline Lost $_{\text {op }}$ & Lost operations \\
\hline Lrem & Level of remaining resources \\
\hline Maint & Maintenance \\
\hline Ntw & No-Wait \\
\hline Prec & Precedence \\
\hline Prmp & Preemptions \\
\hline Reentrant & Reentrant flow \\
\hline Removal & Removal times \\
\hline Skp & Skip stages \\
\hline Stoc_dj & Stochastic due dates \\
\hline $\mathrm{ST}_{\text {sd }}-\mathrm{AS}$ & Sequence-dependent setup and anticipatory \\
\hline $\mathrm{ST}_{\mathrm{sd}}-\mathrm{NS}$ & Sequence-dependent setup and non-anticipatory \\
\hline Stsi-AS & Sequence-independent setup and anticipatory \\
\hline $\mathrm{St}_{\mathrm{si}}-\mathrm{NS}$ & Sequence-independent setup and non-anticipatory \\
\hline Transport & Transport times \\
\hline
\end{tabular}

\subsection{Solution Methods}

Since the pioneering work of Johnson, published in 1954 (JOHNSON, 1954), many solution methods have been developed to solve the scheduling problems in many different types of scheduling environments.

According to Yenisey; Yagmahan (2014), the solution methods for scheduling problems can be categorized into:

- Optimum or exact methods: methods that generate an optimal schedule, according to the performance criterion adopted; mathematical models and specific algorithms are used to solve problems, and obtain an optimal solution, as it adds Pereira (2011); 
INDEPENDENT JOURNAL OF MANAGEMENT \& PRODUCTION (IJM\&P)

- Approximate methods: methods that seek to achieve a feasible solution close to the optimum in a reasonable computational time; can be classified, according to Yenisey; Yagmahan (2014), into heuristic and metaheuristic.

The use of optimum methods is justified when dealing with small problems; the solution for problems, using optimum methods, usually demand high computational time, making the search for optimal solutions not viable (PEREIRA, 2011). Thus, Yenisey; Yagmahan (2014) add that, the optimum methods become inefficient for large problems, since they have many jobs, machines and goals, and are combinatorial optimization problems from NP-hard problems class. Arenales et al. (2007) emphasize that the development of integer programming softwares, such as CPLEX, XPRESS, and LINDO, has improved their ability to solve large problems. The Branch-and-Bound (B\&B) method is the approach, according to Yenisey; Yagmahan (2014), most commonly, used to obtain optimal solutions. Arenales et al. (2007) also points the Branch-and-Cut (B\&C), Gomory, Benders, Dantzig-Wolf, and Lagrangian Relaxation to obtain optimal solutions.

Heuristics are methods that generate a schedule of good quality at a reasonable computational time, with no guarantee of optimality. Solutions obtained by heuristics can be used as an initial solution in improving heuristics and metaheuristics. According to Souza; Moccellin (2000) heuristics can be subclassified into:

- Constructive heuristic: the schedule, adopted as the problem solution, is obtained: i) directly from the ordering of jobs by priority indexes, calculated according to the processing times of the jobs; ii) by sorting the best schedule jobs, from a set of schedules obtained, also using priority indexes associated with the jobs, or; iii) from the successive generation of partial schedules jobs, to obtain a complete schedule through some criterion insertion of jobs, and;

- Improvement heuristic: the schedule, adopted as the problem solution, is obtained from initial solutions that, through some iterative procedure (usually involving exchanges of positions jobs in original schedule), are improved, seeking to achieve a better solution, than the current one, according as the performance criterion adopted. 
- Metaheuristics are procedures that coordinate local search strategies at a higher level, creating a process to avoid local minimum, conducting a search of the most robust solution to a problem (GLOVER; KOCHENBERGER, 2003); although there is no consensus in the literature concerning a standard subclassification of metaheuristic, this can be used (PEREIRA, 2011; OLIVEIRA, 2008; and SERAPIÃO, 2009):

- Metaheuristics of relaxation: "...procedures to solve problems with modifications to the original model, the generated solution provides the solution to the original problem." (PEREIRA, 2011, p. 6). This kind of metaheuristics simplifies the real problem, removing and modifying some restrictions of it (OLIVEIRA, 2008).

- Metaheuristics of neighborhood search: “... procedures that run search spaces, which should be considered at each step, the neighborhood of the solution obtained in the previous interaction." (PEREIRA, 2011, p. 16); e.g., according to Blum; Roli (2003), Dreo et al. (2007) and Yenisey; Yagmahan (2014) are: Greedy Randomized Adaptive Search Procedure (GRASP); Tabu Search (TS); Guided Local Search (GLS), Iterated Local Search (ILS), Local Search (LS), and, Variable Neighborhood Search (VNS).

- Metaheuristics based on evolutionary methods: "...procedures focused on sets of solutions that evolve in this space." (PEREIRA, 2011, p. 16); e.g., according to Dreo et al. (2007), Simon (2013) and Yenisey; Yagmahan (2014) : Genetic Algorithm (GA); Estimation of Distribution Algorithm (EDA); Differential Evolution (DE); Memetic Algorithm (MA); Simulated Annealing (SA); Scarter Search (SS); Artificial Immune System (AIS); Colonial Competitive Algorithm (CCA); .and Harmorny Search Optimization (HSO),

- Metaheurisitcs based on swarm intelligence: procedures based on swarm intelligence include any attempt to design algorithms in order to solve problems inspired by the collective behavior of social insects and other animal societies (BONABEAU; DORIGO; THERAULAZ, 1999). Examples of methods based on swarm intelligence are: Ant Colony Optimization (ACO); Particle Swarm Optimization (PSO); Artificial Bee Colony (ABC); Firefly Algorithm (FA); 
Shuffled Frog-Leaping (SFL); and Bacterial Foraging Optimization (BFO) (SERAPIÃO, 2009 and RUIZ-VANOYE, 2012);

- Hybrids metaheuristics: "...procedures that combine two or more metaheuristics and uses search strategies." (PEREIRA, 2011, p. 16).

Boschetti et al. (2009) adds and emphasizes the use of hybrid methods or matheuristics, algorithms that are developed from the interoperation of metaheuristics and mathematical programming techniques.

\section{RESEARCH METHODOLOGY}

The methods qualitative and quantitative were used in this research. For the purpose, this research was classified as descriptive, explanatory, methodological, and as bibliographical.

The databases used in the literature review were: Compendex; Digital Library of Theses and Dissertations; DOAJ; Emerald; Hindawi, Open J-Gate; IEEE Xplore; Science Direct; Web of Knowledge; Scielo and; Brazilian Digital Library (BDTD); Scirus, and; Scopus. The keywords used were: flow shop; hybrid flow shop; flexible flow shop; multiple machines flow shop; flow shop with multiple machines; biobjective; tri-objective, multi-objective; bicriteria; tricriteria, and; multicriteria. An extensive combination of keywords was also used to identify the published papers. A time limitation has not been established.

For each paper, the following topics were reviewed:

i) The machine set environments in Hybrid Flow Shop considered: identical (ID); uniform (UN); unrelated (UR), or; dedicated (DED);

ii) The performance criteria adopted;

iii) The considered constraint(s);

iv) Objective function used: bicriteria; tricriteria; multicriteria;

v) Solution method(s) developed, in terms of:

- Methods categories: optimum methods; approximate methods;

- Approximate methods classification: heuristic; metaheuristic;

- Heuristics subclassification: constructive; improvement; 
INDEPENDENT JOURNAL OF MANAGEMENT \& PRODUCTION (IJM\&P) http://www.ijmp.jor.br

v. 5, n. 4, October - December 2014 ISSN: 2236-269X

DOI: 10.14807/ijmp.v5i4.242

- Metaheuristics subclassification: neighborhood search; evolutionary; swarm intelligence; hybrids;

- Optimum method type developed;

- Metaheuristic type developed.

Analyses were made based on the number of publications and percentage of occurrence.

In the review, only papers that consider the performance criteria simultaneously, in the same objective function, were reviewed.

\section{MULTICRITERIA HYBRID FLOW SHOP SCHEDULING PROBLEM: LITERATURE REVIEW}

A literature review on the development of solution methods for the bicriteria, and multicriteria scheduling problem was presented by Nagar, Heragu \& Haddock (1995); but, the authors did not identify the publications addressing the Hybrid Flow Shop scheduling problem.

Several papers that address the Hybrid Flow Shop scheduling problem with two or more performance criteria have been identified, however, many of these papers deal with the development and analysis methods for each criterion separately.

In the review, 46 articles that simultaneously consider the performance criteria found: Hayrinen et al. (2000); Liu; Chang (2000); Janiak; Lichteinsten (2001); Gupta et al. (2002); Tang et al. (2002); Lin; Liao (2003); Jungwattanaki et al. (2005); Quadt; Kuhn (2005); Sawik (2005); Akrami; Karimi; Hosseini (2006); Torabi; Fatemi-Ghomi; Karimi (2006); Janiak et al. (2007); Jenabi et al. (2007); Jungwattanaki et al. (2007); Quadt; Kuhn (2007); Sawik (2007); Xuan; Tang (2007); Fakhrzad; Heydari (2008); Jungwattanaki et al. (2008); Khalouli; Ghedjati; Hamzaoui (2008); Mahdavi et al. (2008); Behnamian; Fatemi Ghomi; Zandieh (2009); Davoudpour; Ashrafi (2009); Jungwattanaki et al. (2009); Naderi; Zandieh; Roshanaei (2009); Naderi et al. (2009), Weng; Fujimura (2009a); Weng; Fujimura (2009b); Behnamian; Zandieh; Fatemi Ghomi (2010); Dugardin; Yalaoui; Amodeo (2010); Karimi; Zandieh; Karamooz (2010); Khalouli; Ghedjati; Hamzaoui (2010); Li; Wang; Huo (2010); Rashidi; Jahandar; Zandieh (2010); Behnamian; Zandieh (2011); Cho et al. (2011); Li et al. 
(2011); Mousavi; Zandieh; Amiri (2011); Pereira (2011); Zandieh; Karimi (2011); Han et al. (2012); Weng; Wei; Fujimura (2012); Bozorgirad; Logendran (2013); Ebrahiny; Fatemi Ghomi; Karimi (2013); Fadaei; Zandieh (2013); and Jolai et al. (2013).

In addition to what was related above, Sang (2013) points out problems on the mixed integer programming model to Hybrid Flow Shop scheduling proposed by Behnamian; Zandieh (2011). Tables 3: summarizes the main points reviewed in every paper.

Table 3: Papers that presents solution methods for multicriteria Hybrid Flow Shop scheduling problem - Review Summary

\begin{tabular}{|c|c|c|c|c|c|c|}
\hline \multirow[b]{2}{*}{ Papers } & \multirow[b]{2}{*}{$\begin{array}{c}\text { The machine } \\
\text { set } \\
\text { environments }\end{array}$} & \multirow[b]{2}{*}{$\begin{array}{c}\text { Performance } \\
\text { Criterions }\end{array}$} & \multirow[b]{2}{*}{ Constraints } & \multirow[b]{2}{*}{$\begin{array}{l}\text { Objective } \\
\text { Function }\end{array}$} & \multicolumn{2}{|c|}{ Solution methods } \\
\hline & & & & & $\begin{array}{l}\text { Category and } \\
\text { Classification }\end{array}$ & $\begin{array}{c}\text { Sub } \\
\text { Classification } \\
\text { or Type }\end{array}$ \\
\hline $\begin{array}{l}\text { Hayrinen et } \\
\text { al. (2000) }\end{array}$ & UR & $\begin{array}{l}\sum \mathrm{T}_{\mathrm{j}} ; \sum \mathrm{W}_{\mathrm{j}} ; \\
\mathrm{IBS} ; \mathrm{NF}\end{array}$ & $\begin{array}{l}\mathrm{ST}_{\mathrm{sd}} \mathrm{NS} \\
\text { Lost }_{\mathrm{op}}\end{array}$ & Multicriteria & $\begin{array}{c}- \\
\text { Approximate: } \\
\text { i) Heuristic }\end{array}$ & i) Improvement \\
\hline $\begin{array}{c}\text { Liu; Chang } \\
(2000)\end{array}$ & ID & TST; TSC & $\mathrm{ST}_{\mathrm{sd}}-\mathrm{NS}$ & Bicriteria & - Optimum & $\begin{array}{l}\text { MIP and } \\
\text { Rel_Lag }\end{array}$ \\
\hline $\begin{array}{c}\text { Janiak; } \\
\text { Lichteinsten } \\
\text { (2001) }\end{array}$ & ID & $\begin{array}{c}\sum w_{j} E_{j} ; \sum w_{j} T_{j} ; \\
\sum w_{j} W_{j}\end{array}$ & - & Tricriteria & $\begin{array}{l}\text { - Approximate: } \\
\text { i) Heuristic }\end{array}$ & i) Constructive \\
\hline $\begin{array}{c}\text { Gupta et al. } \\
(2002)\end{array}$ & ID & $\begin{array}{l}\sum \mathrm{E}_{\mathrm{j}} ; \sum \mathrm{T}_{\mathrm{j}} ; \\
\mathrm{C}_{\max } ; \sum \mathrm{C}_{\mathrm{j}} \mathrm{d}_{\mathrm{j}}\end{array}$ & - & Multicriteria & $\begin{array}{l}\text { - Approximate: } \\
\text { i) Heuristic; ii) } \\
\text { Metaheuristic }\end{array}$ & $\begin{array}{l}\text { i) Constructive; } \\
\text { ii) } \\
\text { Neighborhood } \\
\text { Search: LS }\end{array}$ \\
\hline $\begin{array}{l}\text { Tang et al. } \\
\text { (2002) }\end{array}$ & ID & $\sum w_{j} E_{j} ; \sum w_{j} T_{j}$ & $\begin{array}{c}\text { Batch } \\
\text { Prec } \\
\mathrm{ST}_{\mathrm{si}}-\mathrm{NS} \\
\text { Removal }\end{array}$ & Bicriteria & $\begin{array}{l}\text { - Optimum; } \\
\text { - Approximate: } \\
\text { i) Heuristic }\end{array}$ & $\begin{array}{c}\text { MIP and } \\
\text { Rel_Lag/PD } \\
\text { i) Improvement }\end{array}$ \\
\hline $\begin{array}{l}\text { Lin; Liao } \\
\text { (2003) }\end{array}$ & DED & $\Sigma W_{j} ; \quad \Sigma O T$ & $\begin{array}{c}\text { Batch } \\
\mathrm{ST}_{\mathrm{sd}}-\mathrm{NS}\end{array}$ & Bicriteria & $\begin{array}{l}\text { - Approximate: } \\
\text { i) Heuristic }\end{array}$ & i) Improvement \\
\hline $\begin{array}{l}\text { Jungwattanaki } \\
\text { et al. (2005) }\end{array}$ & UR & $\mathrm{C}_{\max } ; \sum \mathrm{U}_{\mathrm{j}}$ & $\mathrm{ST}_{\mathrm{sd}}-\mathrm{NS}$ & Bicriteria & $\begin{array}{l}\text { - Approximate: } \\
\text { i) Heuristic; ii) } \\
\text { Metaheuristic }\end{array}$ & $\begin{array}{l}\text { i) Constructive; } \\
\text { ii) Evolutionary: } \\
\text { GA and SA }\end{array}$ \\
\hline $\begin{array}{l}\text { Quadt; Kuhn } \\
\text { (2005) }\end{array}$ & ID & $\begin{array}{l}\text { TSC; IC; } \\
\sum \mathrm{C}_{\mathrm{j}} \mathrm{U}_{\mathrm{j}} ; \\
\sum \mathrm{F}_{\mathrm{j}} / \mathrm{n}\end{array}$ & Batch & Multicriteria & $\begin{array}{l}\text { - Approximate: } \\
\text { i) Heuristic }\end{array}$ & i) Constructive \\
\hline Sawik (2005) & ID & $\begin{array}{l}\sum \mathrm{U}_{\mathrm{j}} ; \sum_{\mathrm{T}_{\mathrm{max}}} ; \mathrm{TWR} \\
\mathrm{T}\end{array}$ & $\begin{array}{l}\text { Batch } \\
\text { Zbfr } \\
\text { H_Fin }\end{array}$ & Multicriteria & - Optimum & MIP \\
\hline $\begin{array}{l}\text { Akrami; } \\
\text { Karimi; } \\
\text { Hosseini } \\
(2006)\end{array}$ & ID & $\mathrm{TSC} ; \sum \mathrm{F}_{\mathrm{j}} / \mathrm{n}$ & $\begin{array}{c}\text { Batch } \\
\mathrm{ST}_{\mathrm{si}}-\mathrm{NS} \\
\text { Zbfr } \\
\text { H_Fin }\end{array}$ & Bicriteria & $\begin{array}{c}\text { - Optimum } \\
\text { - Approximate: } \\
\text { i) } \\
\text { Metaheuristc }\end{array}$ & $\begin{array}{c}\text { MIP } \\
\text { i) } \\
\text { Neighborhood } \\
\text { Search: TS; } \\
\text { and } \\
\text { Evolutionary: } \\
\text { GA }\end{array}$ \\
\hline $\begin{array}{c}\text { Torabi; } \\
\text { Fatemi- } \\
\text { Ghomi; Karimi } \\
(2006)\end{array}$ & ID & $\begin{array}{l}\text { TST; TC; } \\
\text { WIP; IC }\end{array}$ & $\begin{array}{l}\text { Batch } \\
\mathrm{ST}_{\mathrm{si}}-\mathrm{NS} \\
\text { H_Fin }\end{array}$ & Multicriteria & $\begin{array}{c}\text { - Optimum } \\
\text { - Approximate: } \\
\text { i) } \\
\text { Metaheuristc }\end{array}$ & $\begin{array}{c}\text { MIP } \\
\text { i) Evolutionary: } \\
\text { GA }\end{array}$ \\
\hline $\begin{array}{c}\text { Janiak et al. } \\
\quad(2007)\end{array}$ & ID & $\frac{\sum w_{j} E_{j} ; \sum w_{j} T_{j}}{\sum w_{j} W_{j}}$ & - & Tricriteria & $\begin{array}{c}\text { - Approximate: } \\
\text { i) } \\
\text { Metaheuristc }\end{array}$ & $\begin{array}{c}\text { MIP } \\
\text { i) } \\
\text { Neighborhood } \\
\text { Search :TS; } \\
\text { Evolutionary: }\end{array}$ \\
\hline
\end{tabular}


INDEPENDENT JOURNAL OF MANAGEMENT \& PRODUCTION (IJM\&P)

http://www.ijmp.jor.br

v. 5, n. 4, October - December 2014

ISSN: 2236-269X

DOI: 10.14807/ijmp.v5i4.242

\begin{tabular}{|c|c|c|c|c|c|c|}
\hline & & & & & & $\begin{array}{c}\text { SA; } \\
\text { and Hybrid: } \\
\text { TS/SA }\end{array}$ \\
\hline $\begin{array}{c}\text { Jenabi et al. } \\
(2007)\end{array}$ & UR & TST; CE & $\begin{array}{c}\text { Batch } \\
\text { ST } T_{\text {si }}-N S \\
\text { H_Fin } \\
\text { Skp_Stage }\end{array}$ & Bicriteria & $\begin{array}{l}\text { - Optimum } \\
\text { - Approximate: } \\
\text { i) Heuristic; ii) } \\
\text { Metaheuristc }\end{array}$ & $\begin{array}{l}\text { MIP } \\
\text { i) Constructive } \\
\text { ii) Hybrid: } \\
\text { GA/SA }\end{array}$ \\
\hline $\begin{array}{l}\text { Jungwattanaki } \\
\text { et al. (2007) }\end{array}$ & UR & $\mathrm{C}_{\max } ; \sum \mathrm{U}_{\mathrm{j}}$ & $\mathrm{ST}_{\mathrm{sd}}-\mathrm{NS}$ & Bicriteria & $\begin{array}{l}\text { - Approximate: } \\
\text { i) Heuristic; }\end{array}$ & i) Constructive \\
\hline $\begin{array}{l}\text { Quadt; Kuhn } \\
\quad(2007)\end{array}$ & ID & TSC; $\Sigma F_{j} / n$ & $\begin{array}{c}\text { Batch } \\
\mathrm{ST}_{\text {si }}-\mathrm{AS} \\
\text { Skp_Stage }\end{array}$ & Bicriteria & $\begin{array}{c}\text { - Approximate: } \\
\text { i) } \\
\text { Metaheuristc }\end{array}$ & $\begin{array}{l}\text { i) Evolutionary: } \\
\text { GA }\end{array}$ \\
\hline Sawik (2007) & ID & $\sum \mathrm{U}_{\mathrm{j}} ; \mathrm{CPT}$ & $\begin{array}{l}\text { Zbfr } \\
\text { H_Fin }\end{array}$ & Bicriteria & $\begin{array}{l}\text { - Optimum } \\
\text { - Approximate: } \\
\text { i) Heuristc }\end{array}$ & $\begin{array}{c}\text { MIP } \\
\text { i) Contructive }\end{array}$ \\
\hline $\begin{array}{l}\text { Xuan; Tang } \\
\text { (2007) }\end{array}$ & ID & $\sum w_{j} C_{j} ; \sum w_{j} W_{j}$ & $\begin{array}{c}\mathrm{ST}_{\mathrm{si}} \mathrm{NS} \\
\text { Transport } \\
\text { Lost }_{\mathrm{op}} \\
\text { Prec } \\
\end{array}$ & Bicriteria & - Optimum & Rel_Lag \\
\hline $\begin{array}{l}\text { Fakhrzad; } \\
\text { Heydari } \\
(2008)\end{array}$ & ID & $\sum c_{j} E_{j} ; \sum c_{j} T_{j}$ & $\begin{array}{c}\mathrm{ST}_{\mathrm{si}}-\mathrm{NS} \\
\text { Btlck } \\
\text { Lrem }\end{array}$ & Bicriteria & $\begin{array}{l}\text { - Optimum } \\
\text { - Approximate: } \\
\text { i) Heuristc; ii) } \\
\text { Metaheuristic }\end{array}$ & $\begin{array}{c}\text { MIP } \\
\text { i) Constructive } \\
\text { ii) } \\
\text { Neighborhood } \\
\text { Search: TS; } \\
\text { Evolutionary: } \\
\text { SA; and } \\
\text { Hybrid: SA/TS }\end{array}$ \\
\hline $\begin{array}{l}\text { Jungwattanaki } \\
\text { et al. (2008) }\end{array}$ & UR & $\mathrm{C}_{\max } ; \sum \mathrm{U}_{\mathrm{j}}$ & $\begin{array}{l}\mathrm{ST}_{\text {sd-NS }}-\mathrm{NS} \\
\text { Diff_rj }\end{array}$ & Bicriteria & $\begin{array}{l}\text { - Approximate: } \\
\text { i) Heuristc; ii) } \\
\text { Metaheuristic }\end{array}$ & $\begin{array}{c}\text { i) Constructive } \\
\text { ii) Evolutionary: } \\
\text { GA }\end{array}$ \\
\hline $\begin{array}{c}\text { Khalouli; } \\
\text { Ghedjati; } \\
\text { Hamzaoui } \\
(2008)\end{array}$ & ID & $\sum w_{j} E_{j} ; \sum w_{j} T_{j}$ & - & Bicriteria & $\begin{array}{c}\text { - Approximate: } \\
\text { i) } \\
\text { Metaheuristic }\end{array}$ & $\begin{array}{c}\text { i) Swarm } \\
\text { Intelligence: } \\
\text { ACO }\end{array}$ \\
\hline $\begin{array}{l}\text { Mahdavi et al. } \\
\quad(2008)\end{array}$ & ID & $\sum w_{j} T_{j} ; \sum w_{j} L_{j}$ & $\begin{array}{l}\text { Batch } \\
\mathrm{ST}_{\mathrm{si}}-\mathrm{NS}\end{array}$ & Bicriteria & $\begin{array}{c}\text { - Optimum } \\
\text { - Approximate: } \\
\text { i) } \\
\text { Metaheuristic }\end{array}$ & $\begin{array}{c}\text { MIP } \\
\text { i) Evolutionary: } \\
\text { GA }\end{array}$ \\
\hline $\begin{array}{c}\text { Behnamian; } \\
\text { Fatemi } \\
\text { Ghomi; } \\
\text { Zandieh } \\
\text { (2009a) }\end{array}$ & ID & $\mathrm{C}_{\max } ; \Sigma \mathrm{L}_{\mathrm{j}} ; \sum \mathrm{T}_{\mathrm{j}}$ & $\mathrm{ST}_{\text {sd }}-\mathrm{NS}$ & Tricriteria & $\begin{array}{c}\text { - Approximate: } \\
\text { i) } \\
\text { Metaheuristic }\end{array}$ & $\begin{array}{c}\text { i) Hybrid: } \\
\text { GA/LS/SA/VNS }\end{array}$ \\
\hline $\begin{array}{l}\text { Behnamian; } \\
\text { Zandieh; } \\
\text { Fatemi Ghomi } \\
\text { (2009b) }\end{array}$ & ID & $\Sigma \mathrm{E}_{\mathrm{j}} ; \sum \mathrm{T}_{\mathrm{j}}$ & $\begin{array}{c}\text { Batch } \\
\text { ST } T_{\text {sd }}-N S \\
\text { Window_dj }\end{array}$ & Bicriteria & $\begin{array}{c}\text { - Approximate: } \\
\text { i) } \\
\text { Metaheuristic }\end{array}$ & $\begin{array}{c}\text { i) } \\
\text { Neighborhood } \\
\text { Search: VNS; } \\
\text { and } \\
\text { Evolutionary: } \\
\text { SA; and } \\
\text { Swarm } \\
\text { Intelligence: } \\
\text { PSO }\end{array}$ \\
\hline $\begin{array}{l}\text { Davoudpour; } \\
\text { Ashrafi (2009) }\end{array}$ & ID & $\begin{array}{l}\mathrm{T}_{\max } ; \sum \mathrm{C}_{\mathrm{j}} ; \\
\mathrm{L}_{\max } ; \sum \mathrm{W}_{\mathrm{j}} \mathrm{T}_{\mathrm{j}}\end{array}$ & $\begin{array}{l}\mathrm{ST}_{\text {sd }}-\mathrm{NS} \\
\text { Diff_r } \mathrm{r}_{\mathrm{j}}\end{array}$ & Multicriteria & $\begin{array}{c}\text { - Approximate: } \\
\text { i) } \\
\text { Metaheuristic }\end{array}$ & $\begin{array}{c}\text { i) } \\
\text { Neighborhood } \\
\text { Search: } \\
\text { GRASP }\end{array}$ \\
\hline $\begin{array}{c}\text { Jungwattanaki } \\
\text { et al. (2009) }\end{array}$ & UR & $\mathrm{C}_{\max } ; \sum \mathrm{U}_{\mathrm{j}}$ & $\begin{array}{c}\mathrm{ST}_{\text {sd }}-\mathrm{NS} \\
\text { Skp_Stage }\end{array}$ & Bicriteria & $\begin{array}{l}\text { - Approximate: } \\
\text { i) Heuristc; ii) } \\
\text { Metaheuristic }\end{array}$ & $\begin{array}{c}\text { i) Constructive } \\
\text { ii) } \\
\text { Neighborhood } \\
\text { Search: TS; } \\
\text { and } \\
\text { Evolutionary: } \\
\text { GA and SA. }\end{array}$ \\
\hline
\end{tabular}

[http://creativecommons.org/licenses/by/3.0/us/]

Licensed under a Creative Commons Attribution 3.0 United States License 
INDEPENDENT JOURNAL OF MANAGEMENT \& PRODUCTION (IJM\&P)

http://www.ijmp.jor.br

v. 5 , n. 4, October - December 2014

ISSN: 2236-269X

DOI: 10.14807/ijmp.v5i4.242

\begin{tabular}{|c|c|c|c|c|c|c|}
\hline $\begin{array}{c}\text { Naderi; } \\
\text { Zandieh; } \\
\text { Roshanaei } \\
(2009)\end{array}$ & ID & $\mathrm{C}_{\max } ; \mathrm{T}_{\max }$ & $\mathrm{ST}_{\mathrm{sd}}-\mathrm{NS}$ & Bicriteria & $\begin{array}{c}\text { - Approximate: } \\
\text { i) } \\
\text { Metaheuristic }\end{array}$ & $\begin{array}{l}\text { i) Hybrid: } \\
\text { SA/LS }\end{array}$ \\
\hline $\begin{array}{c}\text { Naderi et al. } \\
(2009)\end{array}$ & ID & $\mathrm{C}_{\max } ; \sum \mathrm{T}_{\mathrm{j}}$ & $\begin{array}{l}\mathrm{ST}_{\text {sd }}-\mathrm{NS} \\
\text { Transport }\end{array}$ & Bicriteria & $\begin{array}{c}\text { - Approximate: } \\
\text { i) } \\
\text { Metaheuristic }\end{array}$ & $\begin{array}{l}\text { i) Hybrid: } \\
\text { SA/LS }\end{array}$ \\
\hline $\begin{array}{l}\text { Weng; } \\
\text { Fujimura } \\
(2009 a) \\
\end{array}$ & UR & $\sum \mathrm{E}_{\mathrm{j}} ; \sum \mathrm{T}_{\mathrm{j}}$ & - & Bicriteria & $\begin{array}{l}\text { - Approximate: } \\
\text { i) Heuristic }\end{array}$ & i) Improvement \\
\hline $\begin{array}{l}\text { Weng; } \\
\text { Fujimura } \\
(2009 \mathrm{~b}) \\
\end{array}$ & ID & $\sum w_{j} E_{j} ; \sum w_{j} T_{j}$ & - & Bicriteria & $\begin{array}{l}\text { - Approximate: } \\
\text { i) Heuristic }\end{array}$ & i) Constructive \\
\hline $\begin{array}{l}\text { Dugardin; } \\
\text { Yalaoui; } \\
\text { Amodeo } \\
(2010) \\
\end{array}$ & ID & BTK; $\mathrm{C}_{\max }$ & $\begin{array}{c}\text { Dom_Estage } \\
\text { Reentrant }\end{array}$ & Bicriteria & $\begin{array}{c}\text { - Approximate: } \\
\text { i) } \\
\text { Metaheuristic }\end{array}$ & $\begin{array}{l}\text { i) Evolutionary: } \\
\text { GA }\end{array}$ \\
\hline $\begin{array}{l}\text { Karimi; } \\
\text { Zandieh; } \\
\text { Karamooz } \\
(2010)\end{array}$ & ID & $\mathrm{C}_{\max } ; \sum \mathrm{T}_{\mathrm{j}}$ & $\begin{array}{c}\text { Batch } \\
\text { ST sd-NS } \\
\text { Skp_Stage }\end{array}$ & Bicriteria & $\begin{array}{c}\text { - Approximate: } \\
\text { i) } \\
\text { Metaheuristic }\end{array}$ & $\begin{array}{l}\text { i) Evolutionary: } \\
\text { GA }\end{array}$ \\
\hline $\begin{array}{l}\text { Khalouli; } \\
\text { Ghedjati; } \\
\text { Hamzaoui } \\
(2010)\end{array}$ & ID & $\sum w_{j} E_{j} ; \sum w_{j} T_{j}$ & - & Bicriteria & $\begin{array}{c}\text { - Approximate: } \\
\text { i) } \\
\text { Metaheuristic }\end{array}$ & $\begin{array}{l}\text { i) Swarm } \\
\text { Intelligence: } \\
\text { ACO }\end{array}$ \\
\hline $\begin{array}{c}\text { Li; Wang; Huo } \\
\text { (2010) }\end{array}$ & ID & $\sum \mathrm{W}_{\mathrm{j}} ; \mathrm{MIT}$ & $\mathrm{ST}_{\mathrm{sd}}-\mathrm{NS}$ & Bicriteria & $\begin{array}{c}\text { - Approximate: } \\
\text { i) } \\
\text { Metaheuristic }\end{array}$ & $\begin{array}{l}\text { i) Evolutionary: } \\
\text { GA }\end{array}$ \\
\hline $\begin{array}{l}\text { Rashidi; } \\
\text { Jahandar; } \\
\text { Zandieh } \\
(2010)\end{array}$ & UR & $\mathrm{C}_{\max } ; \mathrm{T}_{\max }$ & $\begin{array}{l}\mathrm{ST}_{\mathrm{sd}}-\mathrm{NS} \\
\text { Block }\end{array}$ & Bicriteria & $\begin{array}{c}\text { - Approximate: } \\
\text { i) } \\
\text { Metaheuristic }\end{array}$ & $\begin{array}{l}\text { i) Evolutionary: } \\
\text { GA }\end{array}$ \\
\hline $\begin{array}{l}\text { Behnamian; } \\
\text { Zandieh } \\
(2011)\end{array}$ & ID & $\Sigma E_{j} ; \sum T_{j}^{2}$ & $\begin{array}{c}\mathrm{ST}_{\text {sd }}-\mathrm{NS} \\
\mathrm{L} \_\mathrm{WT}\end{array}$ & Bicriteria & $\begin{array}{c}\text { - Optimum } \\
\text { - Approximate: } \\
\text { i) } \\
\text { Metaheuristic }\end{array}$ & $\begin{array}{l}\text { MIP } \\
\text { i) Evolutionary: } \\
\text { CCA }\end{array}$ \\
\hline $\begin{array}{l}\text { Cho et al. } \\
(2011)\end{array}$ & ID & $\mathrm{C}_{\max } ; \Sigma \mathrm{T}_{\mathrm{j}}$ & Reentrant & Bicriteria & $\begin{array}{c}\text { - Approximate: } \\
\text { i) } \\
\text { Metaheuristic }\end{array}$ & $\begin{array}{l}\text { i) Evolutionary: } \\
\text { GA }\end{array}$ \\
\hline Li et al. (2011) & ID & $\mathrm{C}_{\max } ; \Sigma \mathrm{T}_{\mathrm{j}}$ & $S T_{s d}-N S$ & Bicriteria & $\begin{array}{c}\text { - Approximate: } \\
\text { i) } \\
\text { Metaheuristic }\end{array}$ & $\begin{array}{l}\text { i) Evolutionary: } \\
\text { GA }\end{array}$ \\
\hline $\begin{array}{c}\text { Mousavi; } \\
\text { Zandieh; } \\
\text { Amiri (2011) } \\
\end{array}$ & ID & $\mathrm{C}_{\max } ; \sum \mathrm{T}_{\mathrm{j}}$ & $\mathrm{ST}_{\mathrm{sd}}-\mathrm{NS}$ & Bicriteria & $\begin{array}{c}\text { - Approximate: } \\
\text { i) } \\
\text { Metaheuristic } \\
\end{array}$ & $\begin{array}{c}\text { i) } \\
\text { Neighborhood } \\
\text { Search: VNS }\end{array}$ \\
\hline Pereira (2011) & ID & $\sum w_{j} E_{j} ; \sum w_{j} T_{j}$ & $\begin{array}{l}\mathrm{ST}_{\text {sd }}-\mathrm{NS} \\
\text { Diff_r } \mathrm{r}_{\mathrm{j}}\end{array}$ & Bicriteria & $\begin{array}{c}\text { - Optimum } \\
\text { - Approximate: } \\
\text { i) } \\
\text { Metaheuristic }\end{array}$ & $\begin{array}{c}\text { MIP } \\
\text { i) } \\
\text { Neighborhood } \\
\text { Search: ILS; } \\
\text { Evolutionary: } \\
\text { GA; and } \\
\text { Hybrid: GA/LS }\end{array}$ \\
\hline $\begin{array}{c}\text { Zandieh; } \\
\text { Karimi (2011) }\end{array}$ & ID & $\sum \mathrm{w}_{\mathrm{j}} \mathrm{T}_{\mathrm{j}} ; \mathrm{C}_{\max }$ & $\begin{array}{c}\text { Batch } \\
S T_{s d}-N S\end{array}$ & Bicriteria & $\begin{array}{c}\text { - Optimum } \\
\text { - Approximate: } \\
\text { i) } \\
\text { Metaheuristic }\end{array}$ & $\begin{array}{c}\text { MIP } \\
\text { i) Evolutionary: } \\
\text { GA }\end{array}$ \\
\hline $\begin{array}{l}\text { Han et al. } \\
(2012)\end{array}$ & ID & $\sum w_{j} E_{j} ; \sum w_{j} T_{j}$ & - & Bicriteria & $\begin{array}{c}\text { - Approximate: } \\
\text { i) } \\
\text { Metaheuristic }\end{array}$ & $\begin{array}{l}\text { i) Hybrid: } \\
\text { PSO/DE }\end{array}$ \\
\hline $\begin{array}{l}\text { Weng; Wei; } \\
\text { Fujimura } \\
(2012)\end{array}$ & UR & $\sum \mathrm{E}_{\mathrm{j}} ; \sum \mathrm{T}_{\mathrm{j}}$ & Dyn_Arr & Bicriteria & $\begin{array}{l}\text { - Approximate: } \\
\text { i) Heuristic }\end{array}$ & i) Constructive \\
\hline $\begin{array}{l}\text { Bozorgirad; } \\
\text { Logendran } \\
(2013)\end{array}$ & UR & WIP; $\Sigma \mathrm{T}_{\mathrm{j}}$ & $\begin{array}{l}\text { Batch } \\
\text { ST }_{\text {sd }}-\mathrm{NS}\end{array}$ & Bicriteria & $\begin{array}{c}\text { - Optimum } \\
\text { - Approximate: } \\
\text { i) } \\
\text { Metaheuristic }\end{array}$ & $\begin{array}{c}\text { MIP } \\
\text { i) } \\
\text { Neighborhood } \\
\text { Search: TS }\end{array}$ \\
\hline
\end{tabular}


INDEPENDENT JOURNAL OF MANAGEMENT \& PRODUCTION (IJM\&P)

http://www.ijmp.jor.br

v. 5 , n. 4, October - December 2014

ISSN: 2236-269X

DOI: 10.14807/ijmp.v5i4.242

\begin{tabular}{|c|c|c|c|c|c|c|}
\hline & & & & & & \\
\hline $\begin{array}{c}\text { Ebrahiny; } \\
\text { Fatemi } \\
\text { Ghomi; Karimi } \\
(2013) \\
\end{array}$ & ID & $\mathrm{C}_{\max } ; \sum \mathrm{T}_{\mathrm{j}}$ & $\begin{array}{l}\mathrm{ST}_{\mathrm{sd}}-\mathrm{NS} \\
\mathrm{Stoc} \mathrm{d}_{\mathrm{j}}\end{array}$ & Bicriteria & $\begin{array}{c}\text { - Approximate: } \\
\text { i) } \\
\text { Metaheuristic }\end{array}$ & $\begin{array}{l}\text { i) Evolutionary: } \\
\text { GA }\end{array}$ \\
\hline $\begin{array}{c}\text { Fadaei; } \\
\text { Zandieh } \\
(2013)\end{array}$ & ID & $\mathrm{C}_{\max } ; \sum \mathrm{T}_{\mathrm{j}}$ & $\begin{array}{c}\text { Batch } \\
\mathrm{ST}_{\mathrm{sd}}-\mathrm{NS}\end{array}$ & Bicriteria & $\begin{array}{c}\text { - Approximate: } \\
\text { i) } \\
\text { Metaheuristic }\end{array}$ & $\begin{array}{l}\text { i) Evolutionary: } \\
\text { GA }\end{array}$ \\
\hline $\begin{array}{l}\text { Jolai et al } \\
\text { (2013) }\end{array}$ & ID & $\mathrm{C}_{\max } ; \mathrm{T}_{\max }$ & No-wait & Bicriteria & $\begin{array}{c}\text { - Optimum } \\
\text { - Approximate: } \\
\text { i) } \\
\text { Metaheuristic }\end{array}$ & $\begin{array}{c}\mathrm{MIPi}) \\
\text { Evolutionary: } \\
\mathrm{SA}\end{array}$ \\
\hline
\end{tabular}

5. MULTICRITERIA HYBRID FLOW SHOP SCHEDULING PROBLEM: LITERATURE ANALYSES

Methods for the multicriteria Hybrid Flow Shop (HFS) were found in 46 papers.

Figure 3 shows the number of papers published per year, and Figure 4 shows the evolution of research in multicriteria HFS scheduling problem.

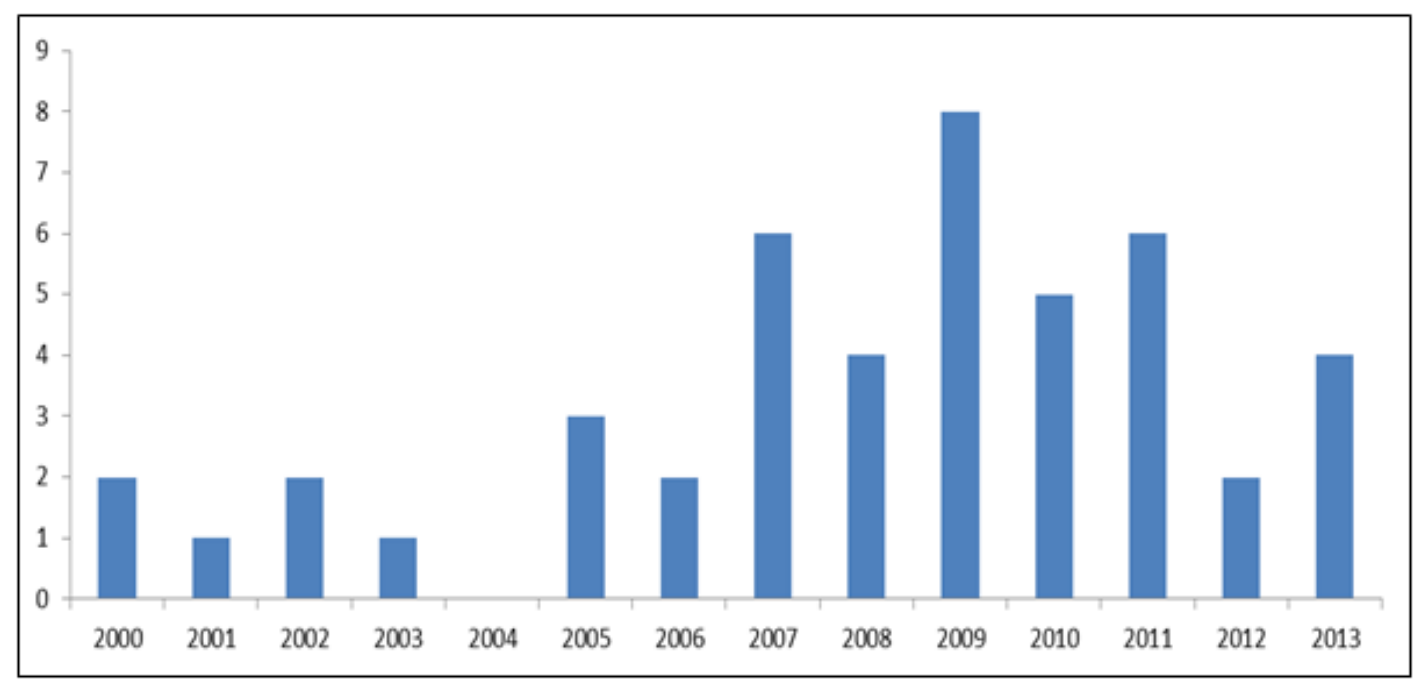

Figure 3: Number of publicarions per year in multicriteria HFS scheduling problem

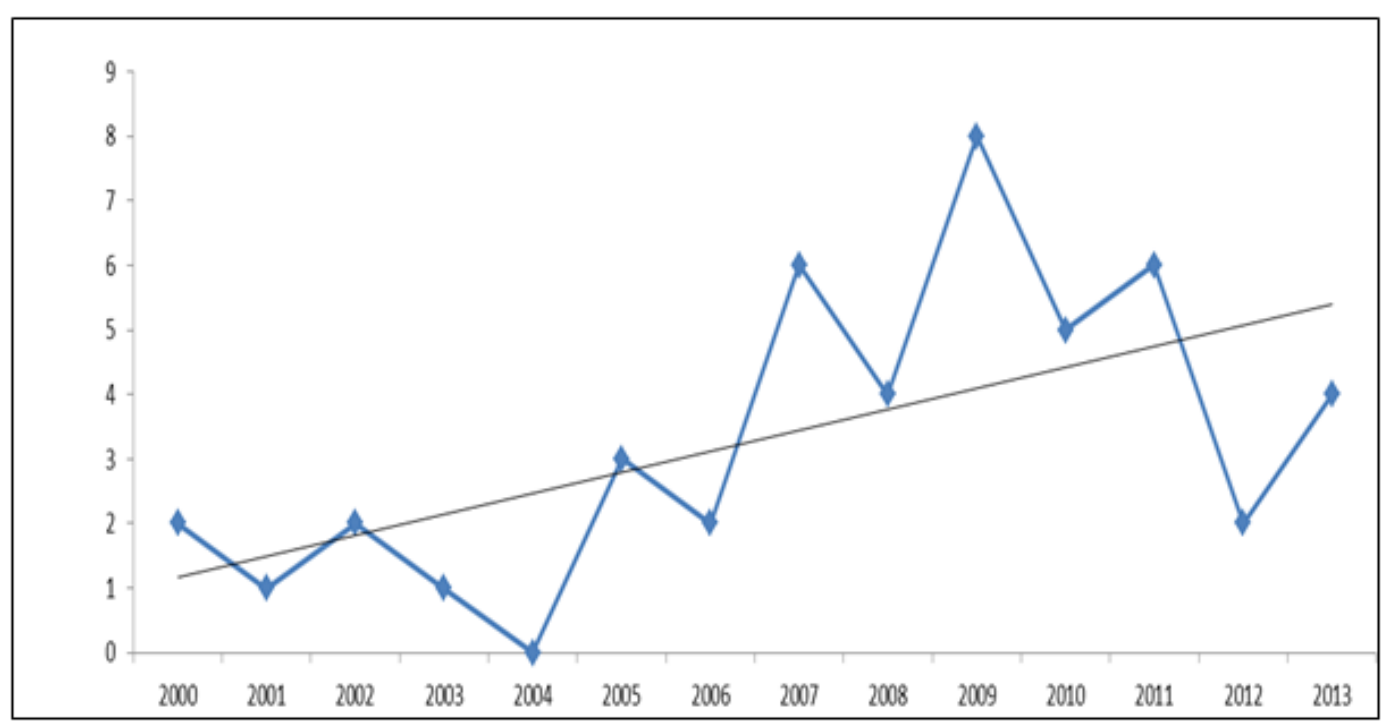

Figure 4: Evolution of research in multicriteria HFS scheduling problem 
INDEPENDENT JOURNAL OF MANAGEMENT \& PRODUCTION (IJM\&P)

http://www.ijmp.jor.br

v. 5, n. 4, October - December 2014

ISSN: 2236-269X

DOI: 10.14807/ijmp.v5i4.242

When analyzing Figure 4, it can be seen that, although in some years the number of publications decreased, there is a growth trend in the researches in multicriteria HFS.

Regarding to the machine set environments in HFS considered, as shown in Figure 5, in most papers (85\%), the identical parallel machines environment is adopted; in 10 (13\%), the unrelated parallel machines environment is adopted, and; in only 1 paper (2\%), the dedicated parallel machines environment is adopted. Figure 5 shows the percentage of use of the different types of parallel machines in developing solution methods for the multicriteria Hybrid Flow Shop.

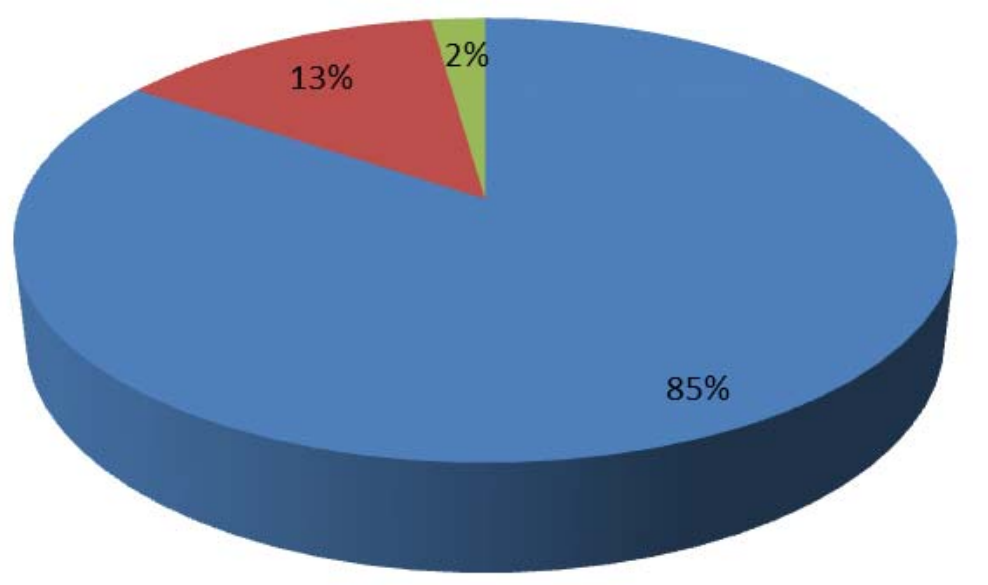

Identical

- Unrelated

- Dedicated

Figure 5: Machines types in parallel used for developing solution methods for multicriteria HFS

Restrictions are present in 38 papers (82.60\%):

- Setup times restrictions are the ones that appears more, being considered in $81.57 \%$ (31 papers) of these papers; dependent and non-anticipatory setup are present in 23 works $(74.19 \%$ of papers with setup times restrictions); while independent and non-anticipatory setup are present in 7 works $(22.59 \%$ of papers with setup times restrictions); of papers with setup times restrictions, only Quadt; Kuhn (2007) investigate the development of solution methods with anticipatory setup;

- Batch sizes and scheduling restrictions are present in 36.84\%;

- Finite horizon scheduling restrictions, in 13.15\%;

- Jobs that can skip stages restrictions are considered in $10.52 \%$; 
INDEPENDENT JOURNAL OF MANAGEMENT \& PRODUCTION (IJM\&P) http://www.ijmp.jor.br

v. 5, n. 4, October - December 2014 ISSN: 2236-269X

DOI: 10.14807/ijmp.v5i4.242

- Limited buffers and different release dates restrictions appear in $7.89 \%$;

- Lost operations, precedence, transport time, and reentrant flow restrictions are present in $5.26 \%$ of papers each;

- Blocking, removal times, no-wait, stochastic due dates, window due dates, remaining level of resources, resource bottleneck, dominant stage, dynamic arrival of jobs, and limited waiting time restrictions were seen in $2.63 \%$ of the papers each.

From the 46 papers, 37 papers (80.43\%) address the development of methods with bicriteria function; 3 papers $(6.52 \%)$ work with the development of methods with a tricriteria function, and; 6 papers $(13.04 \%)$ address the development of methods with multicriteria function.

Concerning the bicriteria functions, performance criteria related to delayed jobs $\left(\Sigma \mathrm{T}_{\mathrm{j}}, \Sigma \mathrm{w}_{\mathrm{j}} \mathrm{T}_{\mathrm{j}}, \mathrm{T}_{\max }, \Sigma \mathrm{U}_{\mathrm{j}}\right.$, and $\left.\Sigma \mathrm{W}_{\mathrm{j}}\right)$, combined with other criteria, appear in 19 papers $(51.35 \%)$; Makespan $\left(C_{\max }\right)$ is present in 16 papers $(43,24 \%)$; the criteria oriented just-in-time scheduling $\left(\sum \mathrm{w}_{\mathrm{j}} \mathrm{E}_{\mathrm{j}}\right.$ and $\sum \mathrm{w}_{\mathrm{j}} \mathrm{T}_{\mathrm{j}}, \sum \mathrm{T}_{\mathrm{j}}$ and $\sum \mathrm{E}_{\mathrm{j}}, \sum \mathrm{c}_{\mathrm{j}} \mathrm{E}_{\mathrm{j}}$, and $\left.\sum \mathrm{c}_{\mathrm{j}} \mathrm{T}_{\mathrm{j}}\right)$ appear in 12 papers (35.29\%); Total Setup Cost (TSC) criteria are present in 3 papers (8.10\%); Total Setup Time (TST) and Mean Flow Time $\left(\sum \mathrm{F}_{\mathrm{j}} / \mathrm{n}\right)$ are adopted in 2 papers each (5.40\%); others criteria, as Bottleneck Utilization Rate (BTK), Capacity Utilization Rate (CPT), Inventory Cost (IC), Overtime (OT), Total Idle Time (MIT), and Work-In-Process (WIP) appear in 1 paper each $(2.70 \%)$.

Regarding the tricriteria functions, earliness, tardiness, and waiting time $\left(\sum w_{j} E_{j}, \quad \sum w_{j} T_{j}\right.$ and $\left.\sum w_{j} W_{j}\right)$ criteria appear in 2 papers simultaneously $(66.66 \%)$; Lateness, makespan, and Tardiness $\left(\mathrm{C}_{\max }, \Sigma \mathrm{L}_{\mathrm{j}}\right.$ and $\left.\Sigma \mathrm{T}_{\mathrm{j}}\right)$ are present in 2 papers simultaneously (33.33\%).

In the papers that adopt a multicriteria function, the following performance criteria are considered: Internal Buffer Size (IBS); Inventory Cost (IC); Lateness Maximum ( $\left.\mathrm{L}_{\max }\right)$; Makespan $\left(\mathrm{C}_{\max }\right)$; Maximum Tardiness ( $\left.\mathrm{T}_{\max }\right)$; Mean Flow Time $\left(\Sigma F_{j} / n\right)$; Number of Families (NF); Number of Late Jobs $\left(\Sigma U_{j}\right)$; Tardy Work Ratio (TWR); Total Completion Time $\left(\Sigma \mathrm{C}_{\mathrm{j}}\right)$; Total Earliness $\left(\Sigma \mathrm{E}_{\mathrm{j}}\right)$; Total Lateness $(\Sigma \mathrm{Lj})$; Total Setup Cost (TSC); Total Setup Time (TCT); Total Tardiness $\left(\Sigma \mathrm{T}_{\mathrm{j}}\right)$; Total Waiting $\left(\Sigma \mathrm{W}_{\mathrm{j}}\right)$; Transport Cost $(\mathrm{TC})$; Weighted Due Date of Jobs $\left(\Sigma \mathrm{c}_{\mathrm{j}} \mathrm{d}_{\mathrm{j}}\right)$; Weighted Number of 
INDEPENDENT JOURNAL OF MANAGEMENT \& PRODUCTION (IJM\&P)

Late Jobs $\left(\sum c_{j} U_{j}\right)$; Weighted Total Tardiness $\left(\sum w_{j} T_{j}\right)$, and; Work-In-Process (WIP). The performance criteria related to delayed or advances of jobs $\left(\Sigma T_{j}, \Sigma w_{j} T_{j}, T_{\max }\right.$, $L_{\max }, \Sigma E_{j}, \Sigma U_{j}$ and $\Sigma W_{j}$ ), combined with others criteria, stand out as the performance criteria most frequently adopted (83.33\%).

Regarding the solution methods developed, in terms of methods categories, it was found that 30 papers $(65.21 \%)$ presents approximate methods; 3 papers $(6.52 \%)$ presents optimum methods, and; $13(28.26 \%)$ presents methods in both categories.

It stands out that, several papers that present methods in both categories, do not address the development of optimum methods. These papers present only mixed integer programming formulations, that are solved by specific solvers (i.e., CPLEX), and their results provide parameters to evaluate the approximate methods developed.

Considering both papers that describe only the approximate methods as those present methods in both categories, the vast majority develop metaheuristics, present in 33 papers $(71.73 \%)$; heuristics appear in 16 (34.78\%).

Concerning the heuristics subclassification, from the 16 papers, that investigates the development of heuristics, 12 papers $(75 \%)$ deal with the development of constructive heuristics, and; $4(25 \%)$ deal with the development of improvement heuristics.

Concerning the metaheuristic subclassification, from the 33 papers that present metaheuristics, 10 papers $(30.30 \%)$ present metaheuristics of neighborhood search; $21(63.63 \%)$ presents metaheuristics based on evolutionary methods; 3 $(9,09 \%)$ presents metaheuristics based on swarm intelligence; and; 8 papers (24.24\%) presents hybrids metaheuristics.

From the papers which present metaheuristics, it was observed that several papers develop more than one type of method; the Genetic Algorithm stands out as one of the methods that is most often adopted, being present in 19 papers $(57.57 \%)$; Simulated Annealing appears in 10 papers (30.30\%); Tabu Search appear in 5 (15.15\%); Local Search in 5 (15.15\%); Variable Neighborhood Search appears in 3 papers (9,09\%); Ant Colony Optimization, and Particle Swarm Optimization appear in 2 papers each (5,88\%), and; Colonial Competitive Algorithm, Differential Evolution, 
INDEPENDENT JOURNAL OF MANAGEMENT \& PRODUCTION (IJM\&P)

http://www.ijmp.jor.br

v. 5, n. 4, October - December 2014

ISSN: 2236-269X

DOI: 10.14807/ijmp.v5i4.242

Greedy Randomized Adaptive Search Procedure, and Iterated Local Search appear in 1 each $(6.06 \%)$.

In relation to papers that address the development of hybrids metaheuristics, solution methods that combine Tabu Search and Simulated Annealing are presented by Janiak et al (2007), and Fakhrzad; Heydari (2008); Local Search combined with Simulated Annealing is presented by Naderi; Zandieh; Roshanaei (2009), and Naderi et al. (2009); Local Search combined with Genetic Algorithm is presented by Pereira (2011); Genetic Algorithm with Simulated Annealing is presented by Jenabi; Fatemi Ghomi; Karimi (2007); Genetic Algorithm with Local Search, Simulated Annealing and Variable Neighborhood Search is presented by Behanamian; Fatemi Ghomi; Zandieh (2009); one solution method that combines Swarm Optimization with Differential Evolution is presented by Han et al. (2012).

Concerning the optimum method type, from the 15 papers, 12 papers (80\%) present and discuss the development of methods based only on programming linear and non-linear mathematical formulations, according to the characteristics of the problems under study; only 3 papers (20\%) present mathematical formulations and discuss the development of methods based on Lagrangian Relaxation and Dynamic Programming.

Figure 6 shows the percentage of papers by solution method(s) developed.

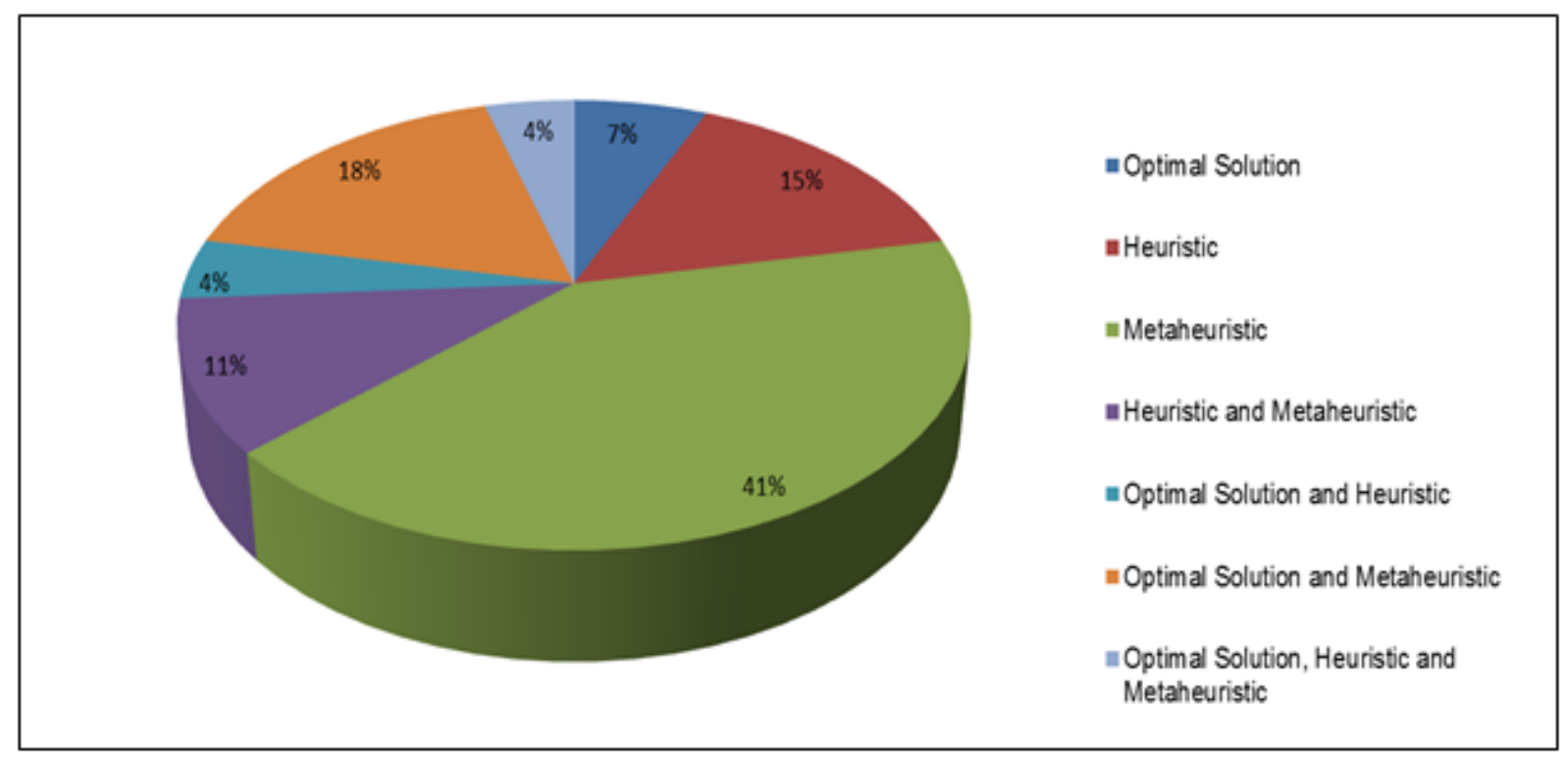

Figure 6: Multicriteria Hybrid Flow Shop scheduling problem - Percentage of papers by solution method(s) developed 
INDEPENDENT JOURNAL OF MANAGEMENT \& PRODUCTION (IJM\&P)

http://www.ijmp.jor.br

v. 5, n. 4, October - December 2014

ISSN: 2236-269X

DOI: 10.14807/ijmp.v5i4.242

\section{CONCLUSIONS}

This article reviews the literature for multicriteria Hybrid Flow Shop (HFS) scheduling problem. 46 articles, published between 2000 and 2013, were found.

The papers were reviewed in terms of the following: the machine set environments in HFS considered; performance criteria adopted; constraint(s) considered; objective function used, and; solution method(s) developed, in terms of methods categories, approximate methods classification, heuristics subclassification, metaheuristic subclassification, optimum method type developed, and metaheuristic type developed.

The analysis on the number of publications over the years shows that there is a trend of growth in the researches in multicriteria HFS scheduling problem. It was not possible to compare the percentage growth in the number of papers published for decades, because the first paper addressing this problem was published in 2000.

Regarding to the machine set environments in HFS considered, identical parallel machine is present in the most papers; only 1 paper treats the HFS scheduling problem with dedicated parallel machines, and; uniform parallel machines are not treated in any article. In practice, with the exception of newly installed plants, the presence of identical parallel machines is not observed with frequency; since the acquisition of machinery in different periods of time, the technological innovations have caused improvements in the capabilities of the same.

The results also showed that, most of the papers are devoted to development of metaheuristics solution methods. Among the metaheuristics, the development of Genetic Algorithms and their variations stands out.

Regarding papers that present heuristics, it was found that constructive heuristics are the focus of a considerable percentage of them.

Among all the papers that present optimum methods, none of them dealt with the development of lower and upper bounds, dominance relationships and search strategies.

In respect of performance criteria the presence of multiple goals in real production environments were considered. Despite the fact that studies, considering more than two performance criteria in the development of solution methods for the 
INDEPENDENT JOURNAL OF MANAGEMENT \& PRODUCTION (IJM\&P)

HFS scheduling problem were found. Only 9 papers (19.56\% of works) adopted three or more performance criteria in the objective function.

The performance criteria related to delayed jobs $\left(\Sigma \mathrm{T}_{\mathrm{j}}, \Sigma \mathrm{w}_{\mathrm{j}} \mathrm{T}_{\mathrm{j}}, \mathrm{T}_{\max }, \Sigma \mathrm{U}_{\mathrm{j}}\right.$ and $\left.\Sigma \mathrm{W}_{\mathrm{j}}\right)$ stands out as one of the performance criteria that are most often adopted. The criteria oriented just-in-time scheduling $\left(\sum \mathrm{w}_{\mathrm{j}} \mathrm{E}_{\mathrm{j}}\right.$ and $\sum \mathrm{w}_{\mathrm{j}} \mathrm{T}_{\mathrm{j}}, \sum \mathrm{T}_{\mathrm{j}}$ and $\sum \mathrm{E}_{\mathrm{j}}, \sum \mathrm{c}_{\mathrm{j}} \mathrm{E}_{\mathrm{j}}$ and $\left.\sum \mathrm{c}_{\mathrm{j}} \mathrm{T}_{\mathrm{j}}\right)$ and were also present in a large number of papers.

Notably, the vast majority of papers include constraints; however, many constraints were not investigated. A new research that considers several constraints is needed, to diminish the gap between the real-world industrial scheduling problems and their treatment in the literature. The most common restrictions are setup times. However, only one paper deals with anticipatory setups.

The analyzes show that, future research may follow different approaches: i) focus on the multicriteria HFS scheduling problems with uniformed and/or dedicated machines in parallel; ii) develop optimum methods to solve multicriteria HFS scheduling problems; iii) develop metaheuristics to solve multicriteria HFS scheduling problems and still not very addressed in literature, such as metaheuristics based on computation evolutionary and swarm intelligence; iv) develop lower bounds and uppers bounds, relations of dominance and different search strategies to obtain solutions for large multicriteria HFS scheduling problems, with reduced computational time; v) investigate the multicriteria HFS scheduling problems with several constraints, present in industries real-world and ignored in the literature, such as anticipatory setups.

\section{REFERENCES}

AKRAMI, B.; KARIMI, B.; MOATTAR-HOSSEINI, S. M. (2006) Two metaheuristic methods for the common cycle economic lot sizing and scheduling in flexible flow shops with limited intermediate buffers: the finite horizon case. Applied

Mathematics and Computation, v.183, n.1, p. 634-645.

ALLAHVERDI, A.; CHENG, T. C. E.; KOVALYOV, M. Y. (2008) A survey of scheduling problems with setup times or costs. European Journal of Operational Research, v. 187, p. 985-1032.

ALLAHVERDI, A.; GUPTA, J. N. D.; ALDOWAISAN, T. (1999) A review of scheduling research involving setup considerations. Omega - The International Journal of Management Science, v. 27, p. 219-239.

ARENALES, M.; ARMENTANO, V.; MORABITO, R.; YANASSE, I. (2007) Pesquisa Operacional. Rio de Janeiro: Elsevier. 
BAKER, K. R. (1974). Introduction to sequencing and scheduling. New York: John Wiley \& Sons, Inc.

BEDWORTH, D. D.; BAILEY, J. E. (1987). Integrated Production Control

Systems: management, analysis, design. 2 ed. New York: John Wiley \& Sons, Inc.

BEHNAMIAN, J.; FATEMI GHOMI, S.M.T.; ZANDIEH, M. (2009a) A Multi-phase covering Pareto-optimal front method to multi-objective scheduling in a realistic hybrid flowshop using a hybrid metaheuristic. Expert Systems with Applications, v. 36, n. 8, p. 11057-11069.

BEHNAMIAN, J.; ZANDIEH, M.; FATEMI GHOMI, S. M. T. (2009b) Due window scheduling with sequence-dependent setup on parallel machines using three hybrid metaheuristic algorithms. International Journal Advanced Manufacture Technology, v. 44, p. 795-808.

BEHNAMIAN, J.; ZANDIEH, M. (2011) A discrete colonial competitive algorithm for hybrid flowshop scheduling to minimize earliness and quadratic tardiness penalties. Expert Systems with Applications, v. 38, p. 14490-14498.

BLUM, C.; ROLI, A. (2003) Metaheuristics in combinatorial optimization: overview and conceptual comparison. ACM Computing Surveys, v. 22, n. 2, p. 241-264.

BOIKO, T. J. P.; MORAIS, M. de F. (2009). The activity of production programming about the operational research view: a theorical conceptual approach. In: VI ENCONTRO TECNOLÓGICO. Proceedings... Campo Mourão: ENTEC, 2009.

BONABEAU, E.; DORIGO, M.; THERAULAZ, G. (1999) Swarm intelligence: from natural to artificial systems, NewYork, Oxford University Press.

BOSCHETTI, M. A.; MANIEZZO, V.; ROFFILLI, M.; ROHLER, A. B. (2009) Metaheuristics: optimization, simulation and control. Lecture Notes in Computer Science, v. 5818.

BOZORGIRAD, M. A.; LOGENDRAN, R. (2013) Bi-criteria group scheduling in hybrid flowshops. International Journal of Production Economics, v. 145, p. 599-612.

BURTSEVA, L.; YAURIMA, V.; PARRA, R. R. (2010) Scheduling methods for hybrid flow shops with setup times. In: Handbook Future Manufacturing Systems. InTech.

CARVALHO, J. D. A. (2000) Production programming. Notes. Sistems and Production department, Engineering School from Minho University. Available in http://pessoais.dps.uminho.pt/jdac/apontamentos/Cap03_Program.pdf.

CHO, H-M.; BAE, S-J., KIM, J.; JEONG, I-J. (2011) Bi-objective scheduling for reentrant hybrid flow shop using Pareto genetic algorithm. Computers \& Industrial Engineering, v. 61, p. 529-541.

DAVOUDPOUR, H.; ASHRAFI, M. (2009) Solving multi-objective SDST flexible flow shop using GRASP algorithm. International Journal Advanced Manufacturing Technology, v. 44, p.737-74.

DREO, J.; AUMASSON,J-P.; TFAILI, W.; SIARRY, P. (2007) Adaptive learning search, a new tool to help comprehending metaheuristics. International Journal on Artificial Intelligence Tools, v. 16. n. 3, p. 483-505. 
DUGARDIN, F.; YALAOUI, F.; AMODEO, L. (2010) New multi-objective method to solve reentrant hybrid flow shop scheduling problem. European Journal of Operational Research, v. 203, p. 22-31.

EBRAHIMINY, S.M.T.; FATEMI GHOMI, S.M.T.; KARIMI, B. (2013) Hybrid flow shop scheduling with sequence dependent family setup time and uncertain due dates. Applied Mathematical Modelling, v. 38, n. 09-10, p. 2490-2504.

FADAEI, M.; ZANDIEH, M. (2013) Scheduling a bi-objective hybrid flow shop with sequence-dependent family setup times using metaheuristics. Arabian Journal of Science Engineering, v. 38, p. 2233-2244.

FAHKRZAD, M. B.; HEYDARI, M. (2008). A heursitc algorithm for hybrid flow-shop production scheduling to miminize the sum of the earliness and tardiness cost. Journal of the Chinese Institute of Industrial Engineers, v. 25, n. 2, p. 105-115.

FRENCH. S. (1982). Sequencing and scheduling: an introduction to the mathematics of the job shop. New York: Wiley.

GLOVER, F.; KOCHENBERGER, G. A. (2003) Handbook of Metaheuristics. Kluwer Academic Publishers, Boston.

GODINHO FILHO, M.; MORAIS, M. F.; BOIKO, T. J. P.; MIYATA, H. H.; VAROLO, F. W. R. (2013). Scheduling in flow shop with sequence-dependent setup times: literature review and analysis. International Journal of Business Innovation and Research, v. 7, n. 4, p. 466-486.

GUPTA, J. N. D.; KRUGER, K.; LAUFF, V.; WERNER, F.; SOTSKOV, Y. N. (2002) Heuristics for hybrid flow shops with controllable processing times and assignable due dates. Computers \& Operations Research, v. 29, p. 1417-1439.

HAN, Z.; SHI, H.; YUAN, W.; ZHANG, Z. (2012) Hybrid PSO/DE solution for the earliness/tardiness case of hybrid flow-shop scheduling problem. In:

INTERNATIONAL CONFERE ON MODELING, IDENTIFICATION AND CONTROL, Wuhan, Proceedings... Wuhan: ICMIC, 2012.

HAYRINEN, T.; JOHNSSON, M.; JOHTELA, T.; SMED, J.; NEVALAINEN. O. (2000) Scheduling algorithms for computer-aided line balancing in printed circuit board assembly. Production Planning and Control, v. 11, n. 5, p. 497-510.

JANIAK, A.; KOZAN, E.; LICHTENSTEIN, M.; OGUZ, C. (2007) Metaheuristic approaches to the hybrid flow shop scheduling problem with a cost-related criterion. International Journal of Production Economics, v. 104, p. 407-424.

JANIAK, A.; LICHTENSTEIN, M. (2001) Comparison of some heuristic algorithms for the flow shop problem with parallel machines to minimize the total earliness, tardiness and waiting time, Operations Research Proceedings, v. $2000-2001$, p. 378-383.

JENABI, M.; GHOMI, S. M. T. F.; TORABI, S. A.; KARIMI, B. (2007) Two hybrid meta-heuristics for the finite horizon ELSP in flexible flow lines with unrelated parallel machines. Applied Mathematics and Computation, v. 186, n.1, p. 230-245.

JOHNSON S. M.; MONTGOMERY D. C. (1974) Operations Research in Production, Planning, Scheduling and Inventory Control. New York: Wiley.

JOHNSON, S. M. (1954) Optimal two- and three-stage production schedules with setup times included. Naval Research Logistics Quarterly, Hoboken, v. 1, p. 61-68. 
JOLAI, F.; ASEFI, H.; RABIEE, M.; RAMEZANI, P. (2013). Bi-objective simulated annealing approaches for no-wait two-stage flexible flow shop scheduling problem. Scientia Iranica, v. 20, n.03, p. 861-872.

JUNGWATTANAKI, J.; REODECHA, M.; CHAOVALITWONGSE, P.; WERNER, F. (2005). An Evaluation of Sequencing Heuristics for Flexible Flowship Scheduling Problems with Unrelated Parallel Machines and Dual Criteria. Magdeburg: Otto Von Guericke, Universität Magdeburg Fakultät für Mathematik. JUNGWATTANAKI, J.; REODECHA, M.; CHAOVALITWONGSE, P.; WERNER, F. (2007) Construtive and Tabu Search for hybrid flow shop problems with unrelated parallel machine and setup times. International Journal of Computational Science, v. 1, n. 2, p. 204-214.

JUNGWATTANAKI, J.; REODECHA, M.; CHAOVALITWONGSE, P.; WERNER, F. (2008). Algorithms for flexible flow shop problems with unrelated parallel machines, setup times, and dual criteria. Introduction Journal Advanced Manufactury Technology, v. 37, p. 354-370.

JUNGWATTANAKI, J.; REODECHA, M.; CHAOVALITWONGSE, P.; WERNER, F. (2009) A comparison of scheduling algorithms for flexible flow shop problems with unrelated parallel machines, setup times, and dual criteria. Computers \& Operations Research, v. 36, n. 2, p. 358-378.

KARIMI, N.; ZANDIEH, M.; KARAMOOZ, H. R. (2010) Bi-objective group scheduling in hybrid flexible flowshop: a multi-phase approach. Expert Systems with Applications, v. 37, p. 4024-4032.

KHALOULI, S.; GHEDJATI, F.; HAMZAOUI, A. (2008). Ant Colony Optimization for solving a bi-criteria hybrid flow shop problem. In: INTERNATIONAL CONFERENCE ON SYSTEMS, MAN AND CYBERNETICS, Singapore. 2008.

Proceedings...Singapore: IEEE/SMC 2008.

KHALOULI, S.; GHEDJATI, F.; HAMZAOUI, A. (2010) A meta-heuristic approach to solve a JIT scheduling problem in hybrid flow shop. Engineering Applications of Intelligence, v.23, p. 765-771.

LI, L., WANG, L.; HUO, J. (2010) Hybrid flowshop scheduling with setup times for cold treating process in Baoshan Iron \& Steel Complex. In: INTERNATIONAL CONFERENCE ON LOGISTICS SYSTEMS AND INTELLIGENT MANAGEMENT, Harbin. Proceedings...Hammamet: IEEE/LSIM, 2011.

LI, X.; CHEHADE, H.; YALAOUI, F.; AMODEO, L. (2011) Lorenz dominance based metaheuristic to solve a hybrid flowshop scheduling problem with sequence dependent setup times. In: INTERNATIONAL CONFERENCE ON COMPUTATION AND CONTROL APPLICATIONS, Hammamet. Proceedings... Hammamet: CCCA, 2011.

LIN, H. T.; LIAO, C. J. (2003) A case study in a two-stage hybrid flow shop with setup time and dedicated machines. International Journal of Production Economics, v. 86, n. 2, p. 133-43.

LIU, C.; CHANG, S. (2000) Scheduling flexible flow shops with sequence-dependent setup effect. Transactions on Robotics and Automation, v. 16, p. 408-419. 
MACCARTHY, B. L.; LIU, J. Y. (1993). Adressing the gap in scheduling research: a review of optimization and heuristic methods in production scheduling. International Journal of Production Research, v. 31, n. 1, p. 59-79.

MAHDAVI, I.; MOJARAD, M.S.; JAVADI, B.; TAJDIN, A. (2008) A genetic approach for solving a hybrid flow shop scheduling problem. In: INDUSTRIAL ENGINEERING AND ENGINEERING MANAGEMENT, Singapore. Procedings... Singapore: IEEM, 2008.

MORAIS, M. F. (2008) Constructive heuristic methods to reduce the stock being processed in hybrid flow shop production environments wirth setup times dependent from sequency. Dissertation (Master's degree) graduation in Production Engeneering, Engeneering school from São Carlos, São Paulo university, São Carlos.

MORAIS, M. F.; GODINHO FILHO, M.; BOIKO, T. J. P. (2013) Hybrid flow shop scheduling problems involving setup considerations: a literature review and analysis. International Journal of Industrial Engineering, v. 20, n.11-12, p. 613-629.

MORAIS, M. F.; MOCCELLIN, J. V. (2010) Constructive heuristics methods to minimizing work in process in environment production hybrid flow shop with asymmetric sequence dependent setup times. Journal of Management and Production, v. 17, n. 2, p. 367- 375.

MORTON, T. E.; PENTICO, D. W. (1993) Heuristic Scheduling Systems. Wiley, New York.

MOUSAVI, S.; ZANDIEH, M.; AMIRI, M. (2011) An efficient bi-objective heuristic for scheduling of hybrid flow shops. The International Journal of Advanced Manufacturing Technology, v. 54, n. 1-4, p. 287-307.

NADERI, B.; ZANDIEH, M.; BALAG, A.K.G.; ROSHANAEI, V. (2009) An improved simulated annealing for hybrid flowshops with sequence-dependent setup and transportation times to minimize total completion time and total tardiness. Expert systems with Applications, v.36, n.6, p. 9625-9633.

NADERI, B.; ZANDIEH, M.; ROSHANAEI, V. (2009) Scheduling hybrid flowshops with sequence dependent setup times to minimize makespan and maximum tardiness. International Journal Advanced Manufacturing Technology, v. 41, p.1186-1198.

NAGAR, A.; HADDOCK, J.; HERAGU, S. (1995) Multiple and bicritério scheduling: a literature survey. European Journal of Operational Research, v. 81, p.88-104.

OLIVEIRA, R. B. (2008) Development of a multi-agent architecture based on methaheuristics with an adaptive learning search approach. Dissertation (master's degree). Graduation in Computational and mathematical modeling, Federal Center of Technological Education, Minas Gerais, Belo Horizonte.

PEREIRA, A. M. S. (2011) Metaheuristics for the flowshop flexible with advance and tardiness problem. Dissertation (Master's degree) graduation in computational science, Federal University from Viçosa, Viçosa.

PINEDO, M. (2008) Theory, Algorithms and Systems. New Jersey: Prentice-Hall. 
QUADT, D.; KUHN, H. (2005) Conceptual framework for lot-sizing and scheduling of flexible flowlines. International Journal of Production Research, v. 43, n. 11, p. 2291-308.

QUADT, D.; KUHN, H. (2007) Batch scheduling of jobs with identical process times on flexible flowlines. International Journal of Production Economics, v. 105, n. 2, p. 385-401.

RASHIDI, E.; JAHANDAR, M.; ZANDIEH, M. (2010) An improved hybrid multiobjective parallel genetic algorithm for hybrid flow shop scheduling with unrelated parallel machines. International journal advanced manufacturing technology, v. 49, n. 9-12, p. 1129-1139.

RUIZ-VANOYE, J.; DÍAZ-PARRA, O.; COCÓN, F.; SOTO, A. (2012) Meta-heuristics algorithms based on the grouping of animals by social behavior for the traveling salesman problem. International Journal of Combinatorial Optimization Problems and Informatics, v. 3, n. 3, p.104-123.

SANG, H-Y. (2013) Erratum to "A discrete colonial competitive algorithm for hybrid flowshop scheduling to minimize earliness and quadric tardiness penalties"[Expert Syst. Appl. 38 (2011) 14490-14498]. Expert Systems with Applications, v. 40, p. 4270-4272.

SAWIK, T. (2005) Integer programming approach to production scheduling for maketo-order manufacturing. Mathematical and Computer Modelling, v. 41, n. 1, p. 99118.

SAWIK, T. (2007) A lexicographic approach to bi-objective scheduling of singleperiod orders in make-to-order manufacturing. European Journal of Operational Research, v.180, p.1060-1075.

SERAPIÃO, A. B. S. (2009) Optimization fundamentals by swarm inteligence: a global view Contrlole \& Automação journal, v. 20, n. 3, p.271-304.

SETHANAN, K. (2001) Scheduling flexible flowshops with sequence dependent setup times. These (Doctored). College of Engineering and Mineral Resources, West Virginia University, Morgantown.

SIMON, D. (2013) Evolutionary optimization algorithms: biologically inspired and population-based approaches to computer intelligence. New York: John Wiley \& Sons, Inc.

SOUZA, A. B. D.; MOCCELLIN, J. V. (2000) Hybrid metaheuristic genetic algorithm Busca-Tabu for flow shop operations programming.In: SIMPÓSIO BRASILEIRO DE PESQUISA OPERACIONAL, XXXII, Rio de Janeiro, Proceedings... Rio de Janeiro: SBPO, 2000.

TANG, L.; LUH, P. B.; LIU, J.; FANG, L. (2002) Steel-making process scheduling using Lagrangian relaxation. International Journal of Production Research, v. 40, n. 1 , p. 55-70.

TAVAKKOLI-MOGHADDAM, R.; RAHIMI-VAHED, A.; MIRZAEI, A. (2007) A hybrid multi-objective immune algorithm for a flow shop scheduling problem with bi-objectives: Weighted mean completion time and weighted mean tardiness. Information Sciences, v. 177, p. 5072-5090. 
TORABI, S. A.; FATEMI-GHOMI, S. M. T.; KARIMI, B. (2006) A hybrid genetic algorithm for the finite horizon economic lot and delivery scheduling in supply chains. European Journal of Operational Research, v. 173, p.173-189.

WENG, W.; FUJIMURA, S. (2009a) Online Scheduling of Flexible Flow Shop Manufacturing. In: INTERNATIONAL JOINT CONFERENCE ON COMPUTATIONAL SCIENCES AND OPTIMIZATION, Sanya, Hainan, China. Proceedings... Sanya, Hainan, China.: IJCCSO, 2009. 978-0-7695-3605-7/09.

WENG, W.; FUJIMURA, S. (2009b) Distributed Feedback Mechanism for Just-InTime Scheduling Problem. IN: INTERNATIONAL CONFERENCE ON COMPUTER AND INFORMATION SCIENCE, 8, Shanghai, China. Proceedings... Shanghai, China: Eigth IEEE/ACIS, 2009.

WENG, W.; WEI, X.; FUJIMURA, S. (2012) Dynamic routing strategies for JIT production in hybrid flow shops. Computers \& Operations Research, v. 39, p. 3316-3324.

XUAN, H; TANG, L. X. (2007) Scheduling a hybrid flow shop with batch production at the last stage. Computers \& Operations Research, v. 34, n. 9, p. 2718-33.

YANG, W.; LIAO, C. (1999) Survey of scheduling research involving setup times. International Journal of Systems Science, Abington, v. 30, n. 2, p. 143-155.

YENISEY, M. M.; YAGMAHAN, B. (2014) Multi-objective permutation flow shop scheduling problem: Literature review, classification and current trends. Omega, v. 45, p. 119-135.

ZANDIEH, M.; KARIMI, N. (2011) An adaptive multi-population genetic algorithm to solve the multi-objective group scheduling problem in hybrid flexible flowshop with sequence-dependent setup times. Journal of Intelligence Manufacturing, v. 22, p. 979-989. 